\title{
Predictive In Vitro-In Vivo Extrapolation for Time Dependent Inhibition of CYP1A2, CYP2C8, CYP2C9, CYP2C19, and CYP2D6 Using Pooled Human Hepatocytes, Human Liver Microsomes, and a Simple Mechanistic Static Mode ${ }^{[\Phi}$
}

\author{
Diane Ramsden, ${ }^{1}$ Elke S. Perloff, ${ }^{1}$ Andrea Whitcher-Johnstone, Thuy Ho, Reena Patel, \\ Kirk D. Kozminski, Cody L. Fullenwider, and J. George Zhang \\ Takeda Development Center Americas, Inc., Cambridge, Massachusetts (D.R.); Corning Gentest Contract Research Services, \\ Corning Life Sciences, Woburn, Massachusetts (E.S.P., T.H., R.P., J.G.Z.); Takeda Development Center Americas, Inc., San \\ Diego, California (K.D.K., C.L.F.); and Boehringer Ingelheim Pharmaceuticals Inc., Ridgefield, Connecticut (A.W.-J.)
}

Received October 7, 2021; accepted November 12, 2021

\section{ABSTRACT}

Inactivation of Cytochrome P450 (CYP450) enzymes can lead to significant increases in exposure of comedicants. The majority of reported in vitro to in vivo extrapolation (IVIVE) data have historically focused on CYP3A, leaving the assessment of other CYP isoforms insubstantial. To this end, the utility of human hepatocytes (HHEP) and human liver microsomes (HLM) to predict clinically relevant drug-drug interactions was investigated with a focus on CYP1A2, CYP2C8, CYP2C9, CYP2C19, and CYP2D6. Evaluation of IVIVE for CYP2B6 was limited to only weak inhibition. A search of the University of Washington DrugDrug Interaction Database was conducted to identify a clinically relevant weak, moderate, and strong inhibitor for selective substrates of CYP1A2, CYP2C8, CYP2C9, CYP2C19, and CYP2D6, resulting in 18 inhibitors for in vitro characterization against 119 clinical interaction studies. Pooled human hepatocytes and HLM were preincubated with increasing concentrations of inhibitors for designated timepoints. Time dependent inhibition was detected in HLM for four moderate/ strong inhibitors, suggesting that some optimization of incubation conditions (i.e., lower protein concentrations) is needed to capture weak inhibition. Clinical risk assessment was conducted by incorporating the in vitro derived kinetic parameters maximal rate of enzyme inactivation $\left(\mathrm{min}^{-1}\right)\left(k_{\text {inact }}\right)$ and concentration of inhibitor resulting in $50 \%$ of the maximum enzyme inactivation $\left(K_{1}\right)$ into static equations recommended by regulatory authorities. Significant overprediction was observed when applying the basic models recommended by regulatory agencies. Mechanistic static models, which consider the fraction of metabolism through the impacted enzyme, using the unbound hepatic inlet concentration lead to the best overall prediction accuracy with $92 \%$ and $85 \%$ of data from HHEPs and HLM, respectively, within twofold of the observed value.

\section{SIGNIFICANCE STATEMENT}

Coupling time-dependent inactivation parameters derived from pooled human hepatocytes and human liver microsomes (HLM) with a mechanistic static model provides an easy and quantitatively accurate means to determine clinical drug-drug interaction risk from in vitro data. Optimization is needed to evaluate time-dependent inhibition (TDI) for weak and moderate inhibitors using HLM. Recommendations are made with respect to input parameters for in vitro to in vivo extrapolation (IVIVE) of TDI with non-CYP3A enzymes using available data from HLM and human hepatocytes.
This paper received no external funding.

All authors are employed by their respective noted employers and are provided compensation and may also be stockholders. The authors are responsible for the content contained in this article and have no actual or perceived conflict of interest with its contents.

${ }^{1}$ D.R. and E.S.P. contributed equally to this work

dx.doi.org/10.1124/dmd.121.000718.

S This article has supplemental material available at dmd.aspetjournals.org.

\section{Introduction}

Evaluating the potential for a drug candidate to inactivate Cytochrome P450 (CYP450) enzymes is important to predict the likelihood of clinically relevant drug-drug interactions (DDIs). Enzyme inactivation is a process whereby, during the catalytic cycle of an enzyme, a reactive intermediate is produced that binds to and irreversibly inhibits the active site of that enzyme. Competitive inhibition and inactivation are mechanistically distinct processes: in the case of competitive inhibition,

ABBREVIATIONS: $\mathrm{C}_{\max , \mathrm{ss}}$, maximal observed concentration in blood at steady state; $\mathrm{C}_{\max , \mathrm{ss}, \mathrm{u}}$, maximal unbound plasma concentration at steady state; CYP, cytochrome P450; DDI, drug-drug interaction; EMA, European Medicines Agency; FDA, Food and Drug Administration; $F_{m}$, fraction metabolized through the pathway; FN, false negatives; GMFE, geometric mean fold error; HHEP, human hepatocytes; HLM, human liver microsomes; $I_{\text {inlet,max }, u}$, unbound hepatic inlet concentration; IVIVE, in vitro to in vivo extrapolation; $\mathrm{K}_{\mathrm{i}}$, inhibition constant for reversible inhibition; $\mathrm{k}_{\text {inact }}$, maximal rate of enzyme inactivation $\left(\mathrm{min}^{-1}\right) ; \mathrm{K}_{\mathrm{l}}$, concentration of inhibitor resulting in $50 \%$ of the maximum enzyme inactivation; $\mathrm{k}_{\mathrm{obs}}$, first order rate constant for inactivation estimated from the slope of residual activity (In) versus preincubation time at each inhibitor concentration $\left(\mathrm{min}^{-1}\right) ; \mathrm{k}_{\text {solvent }}$, first order rate constant for inactivation estimated from the slope of residual activity (In) versus preincubation time for the solvent control [ $\mathrm{min}^{-1}$ ] (may be constrained to equal the $\mathrm{k}_{\mathrm{obs}}$ for the vehicle control); LC-MS/MS, liquid chromatography tandem mass spectrometry; In, natural log residual activity; MM, Michaelis-Menten; MSM, mechanistic static model; PBPK, Physiologically-Based Pharmacokinetic; RMSE, root mean square error; PMDA, Pharmaceutical and Medical Devices Agency; TDI, time-dependent inhibition; UW-DIDB, University of Washington Drug-Drug Interaction Database; WME, William's medium E. 
enzyme activity is restored when the inhibitor is removed, whereas removal of the inactivator does not restore the activity of the inactivated enzyme. Consequently, recovery of enzyme activity depends on the rate of enzyme resynthesis. Regulatory agencies have provided industry guidance on the conduct of in vitro studies to predict the potential of a drug to mediate DDI through enzyme inactivation [European Medicines Agency (EMA), Food and Drug Administration (FDA) and Pharmaceutical and Medical Devices Agency (PMDA)]. Currently, human liver microsomes (HLM) or recombinantly expressed enzymes are the in vitro systems most often used to evaluate CYP450 inactivation (Grimm et al., 2009); however, although HLM have been successfully used to predict clinically relevant DDIs for known CYP450 inactivators (Obach et al., 2007), there is a tendency to overpredict clinical DDIs (Chen et al., 2011). As with any in vitro system, the environment of the typical microsomal and recombinant enzyme assays differs significantly from the in vivo environment that they strive to model. Consequently, assumptions are made about a drug, for example, complete permeability across biologic membranes, minimal binding to microsomal proteins, and minimal contribution of non-CYP450 metabolism. By using a more physiologically complete system such as human hepatocytes, which have an intact plasma membrane, functional membrane transporters, a complete set of hepatic CYP450 and non-CYP450 enzymes, some assumptions associated with HLM and recombinant systems may no longer be necessary. Although human hepatocytes can be used to evaluate the potential for CYP450 inactivation, there are only a few peer-reviewed examples, prompting the need for additional research as a prerequisite for more routine use of this model. Nevertheless, human hepatocytes could provide mechanistic insight that supports conventional inactivation studies where non-CYP450 metabolites are generated or significant intracellular accumulation of a drug is suspected. In human hepatocytes, active transport of a drug into or out of cells can affect the concentration of a drug present at the CYP450 active site, ultimately affecting the inactivation parameters determined. $\mathrm{Xu}$ et al. and Chen et al. postulated that differences in inactivation parameters between human hepatocytes and HLM were due to active transport of drugs in hepatocytes (Xu et al., 2009; Chen et al., 2011). A decrease in active uptake of a compound with limited permeability will decrease the inactivation potential by decreasing the concentration of a drug at the site of inactivation. Conversely, a decrease in active efflux will increase the inactivation potential by increasing the amount of a drug available for metabolism (Lam et al., 2006). Indeed, erythromycin, diltiazem, and troleandomycin are known or suspected substrates of membrane transporters and result in the greatest discrepancy in inactivation parameters between HLM and hepatocytes (Seelig and Landwojtowicz, 2000; Kostrubsky et al., 2003; Kurnik et al., 2006). Additionally, CYP450 inactivators subject to extensive nonCYP450 metabolism, for example, glucuronidation in vivo, may be mistakenly determined to be clinically relevant inactivators when evaluated using HLM. For example, ezetimibe, a cholesterol-lowering drug, displayed potent in vitro inactivation of CYP3A4 using HLM but did not result in clinically meaningful inhibition, likely due to its extensive glucuronidation (Parkinson et al., 2010). Alternatively, a drug that is significantly metabolized via a non-CYP450 pathway and forms a metabolite that inactivates CYP450 enzymes cannot be detected in conventional microsomal CYP450 inactivation assays, as was observed with an aldehyde oxidase metabolite (Zetterberg et al., 2016). This was also the case with gemfibrozil, which led to significant CYP2C8 DDIs subsequently revealed to be mediated by its major metabolite gemfibrozil-1-O- $\beta$-glucuronide, the potential for DDIs could have been detected if the inactivation studies were initially performed using human hepatocytes (Ogilvie et al., 2006; Parkinson et al., 2010). Although IVIVE efforts for time-dependent inhibition (TDI) have focused primarily on CYP3A inactivation (Eng et al., 2020), it is unclear whether the recommendations made for CYP3A inactivators may translate to other major CYPs or whether hepatocytes could also be a predictive in vitro model to assess TDI of other CYP isoforms. The purpose of the work described here was to assess suspended hepatocytes and HLM as tools for predicting DDI caused by TDI of CYPs 1A2, 2C8, 2C9, 2C19, and 2D6.

\section{Materials and Methods}

\section{Chemicals and Reagents}

Cryopreserved Hepatocyte Recovery Medium was purchased from Life Technology (cat \# CM7000, Carlsbad, CA). Pooled 200 donor mixed gender human liver microsomes were purchased from XenoTech (cat\# H2610, Kansas City, KS). Cimetidine, ciprofloxacin, clopidogrel, dronedarone, fluconazole, fluvoxamine,

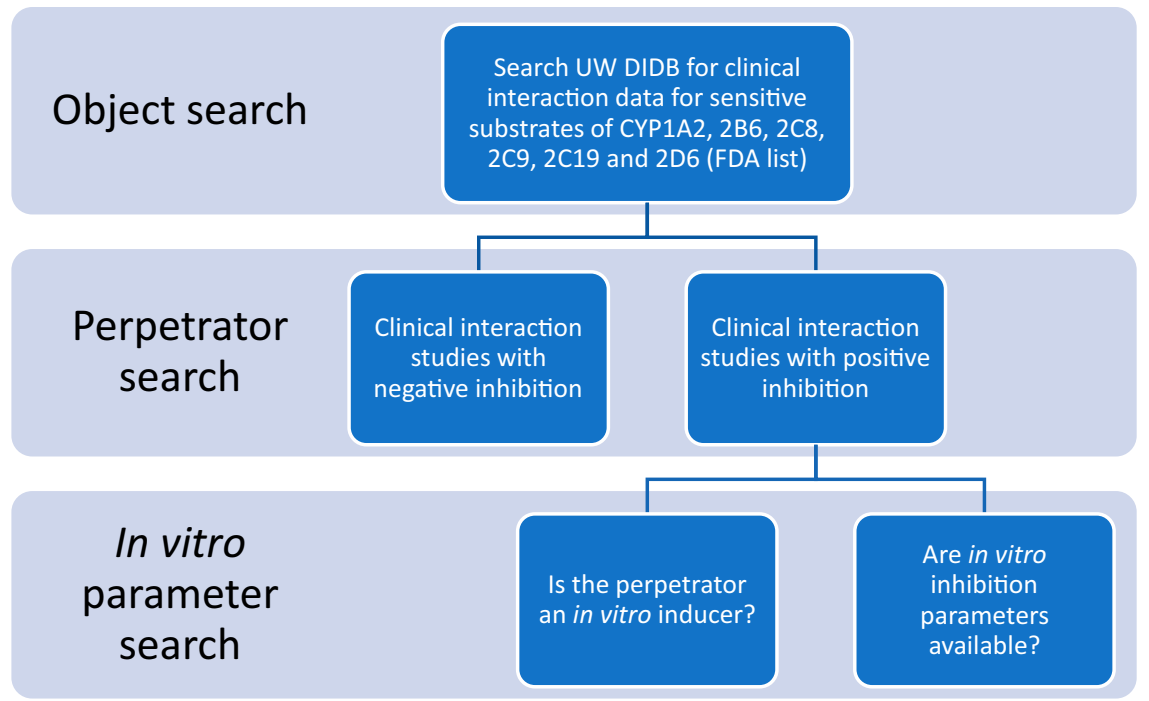

Fig. 1. The workflow for identifying clinically relevant inhibitors included searching the UW-DIDB for clinical data with sensitive objects of CYPs 1A2, 2B6, 2C8, $2 \mathrm{C} 9,2 \mathrm{C} 19$, and 2D6. The data were collated for both positive and negative inhibition, and perpetrators were categorized as negative, weak, moderate, or strong inhibitors dependent on the magnitude of AUC change. Where negative inhibition was defined as AUCR between 1.0- and 1.25-fold, weak was between 1.25- and 2.0-fold AUCR, moderate between 2.0- and 5.0-fold AUCR and strong >5.0-fold AUCR. Once perpetrators were identified, literature searches were performed for existing in vitro data, including inhibition and time-dependent inhibition, induction and transporter substrate, or inhibition observations. 
TABLE 1

Enzyme reaction conditions

\begin{tabular}{|c|c|c|c|c|c|c|}
\hline \multirow[b]{2}{*}{ CYP Isoform } & \multicolumn{2}{|c|}{ P450 Probe Substrate } & \multicolumn{2}{|c|}{ Substrate Concentration $(\mu \mathrm{M})$} & \multicolumn{2}{|c|}{ Incubation Time (min) } \\
\hline & HHEP & HLM & HHEP & HLM & HHEP & HLM \\
\hline CYP1A2 & Phenacetin & Phenacetin & 100 & 180 & 30 & 8 \\
\hline CYP2C8 & Amodiaquine & Paclitaxel & 100 & 40 & 10 & 12 \\
\hline CYP2C9 & Diclofenac & Diclofenac & 100 & 36 & 10 & 8 \\
\hline CYP2C19 & s-Mephenytoin & s-Mephenytoin & 100 & 225 & 30 & 8 \\
\hline CYP2D6 & Dextromethorphan & Dextromethorphan & 25 & 36 & 10 & 8 \\
\hline
\end{tabular}

HLM final protein concentration $(1 \mathrm{mg} / \mathrm{mL})$.

miconazole, moclobemide, omeprazole, paroxetine, tasisulam, ticlopidine trimethoprim, NADPH, and William's medium E were purchased from Millipore Sigma (St. Louis, MO). Mirabegron was obtained from MyBioSource (San Diego, CA), osilodrostat from Selleck Chemicals (Houston, TX), and gemfibrozil from Toronto Research Chemicals (Toronto, ON).

\section{Hepatocytes}

Experiments were performed using cryopreserved human hepatocytes (Cat. \# 454427, Corning Life Sciences, Woburn, MA) pooled from three donors (lot \# 305, 346, and 347, except for ticlopidine, which used lot \#305, 289, and 293). Donor demographics are displayed in Supplemental Table 1.

\section{Methods}

Identification of Clinically Relevant Inhibitors for IVIVE Analysis. Clinical data were collected according to Fig. 1, by searching the University of Washington Drug-Drug Interaction Database (UW-DIDB) for published studies with and without observed changes in area under the curve (AUC) or clearance for sensitive substrates of CYP1A2, CYP2B6, CYP2C8, CYP2C9, CYP2C19, and CYP2D6, indicated on the FDA website (https://www.fda.gov/drugs/ drug-interactions-labeling/drug-development-and-drug-interactions-table-substratesinhibitors-and-inducers), Supplemental Table 2. Clinical data were reviewed to select for multiple dose studies and to identify weak, moderate, and strong inhibitors toward each enzyme. Inhibitors with multiple studies against multiple substrate drugs were prioritized to expand the dataset available for IVIVE analysis and evaluate trends across selective substrates (Table 4). Once clinically relevant inhibitors were identified, the UW-DIDB was searched for in vitro inhibition and induction parameters and for transporter substrate and inhibitor liabilities. Additionally, inhibitor properties including dose level used, maximal observed concentration in blood at steady state $\left(\mathrm{C}_{\mathrm{max}, \mathrm{ss}}\right), \mathrm{Fu}$, $\mathrm{Ka}, \mathrm{Fa}, \mathrm{Fg} \mathrm{Rb}$, and $\log \mathrm{P}$ or $\mathrm{D}$ were collected where possible. The $\mathrm{C}_{\text {max,ss }}$ values, published solubility, and historical in vitro inhibition data were used to identify relevant in vitro test concentrations with the goal being to span concentrations that would enable estimation of the kinetic parameters but limit the likelihood to observe toxicity.

Compilation of In Vitro Parameters from Literature. To expand the analysis to understand whether the recommendations identified for improving the prediction of TDI for CYPs $1 \mathrm{~A} 2,2 \mathrm{C} 8,2 \mathrm{C} 9,2 \mathrm{C} 19$, and $2 \mathrm{D} 6$ could be extended to data generated in HLM, an attempt was made to collate time-dependent inhibition parameters from literature. This was accomplished by searching the UWDIDB for inhibition parameters derived from either human hepatocytes or HLM and analysis was extended to CYP2B6. Data compiled from literature are presented in Supplemental Table 7. Additionally, HLM incubations were conducted within Takeda, using standard experimental conditions for inhibitors with no published data.

Experimental. Hepatocyte incubations. Hepatocytes from three individual lots (reference Supplemental Table 1) were thawed and pooled in Cryopreserved Hepatocyte Recovery Medium, then centrifuged at $100 \mathrm{~g}$ for 10 minutes at room temperature. The supernatant was aspirated, and the pellet was washed with William's medium $\mathrm{E}$ (WME) followed by centrifugation at $40 \mathrm{~g}$ for 3 minutes. Hepatocytes were then resuspended in prewarmed WME at a density of $1.1 \times 10^{6}$ cells $/ \mathrm{mL}$ and $45 \mu \mathrm{l}$ of the cell suspension was loaded into a prewarmed 96 -well plate and equilibrated for 15 minutes at $37^{\circ} \mathrm{C}$. Pretreatment with model compounds were initiated by the addition of $5 \mu \mathrm{l}$ of prewarmed 10X inhibitor working solution in WME. The final concentration of DMSO in the preincubation was $0.1 \%$. After each preincubation time point, the probe substrate reaction was initiated by addition of $150 \mu \mathrm{l}$ of prewarmed WME containing the substrate (Table 1). At the end of the incubation time (Table 1), the enzyme reaction was stopped by addition of $50 \mu \mathrm{l}$ stop solution $(0.1 \%$ formic acid in acetonitrile containing a stable-isotope labeled internal standard). The plate was stored at $-20^{\circ} \mathrm{C}$ as needed until analysis. The concentrations of probe substrate metabolite formed

TABLE 2

Inhibitors and preincubation conditions

\begin{tabular}{lllcr}
\hline CYP Isoform & Inhibitor & Clinical Inhibition & Concentration Range in Preincubation $(\mu \mathrm{M})$ & Preincubation Times $($ min $)$ \\
\hline CYP1A2 & Cimetidine & Weak & $16-2000$ & $0,15,30,45,60$ \\
CYP1A2 & Ciprofloxacin & Moderate & $3.9-500$ & $0,15,30,45,60$ \\
CYP1A2 & Fluvoxamine & Strong & $0.0046-10$ & $1.5-200$ \\
CYP2C8 & Trimethoprim & Weak & $0.3-600$ & $0,5,10,15,20$ \\
CYP2C8 & Clopidogrel & Moderate & $0.1-300$ & $0,15,30,45,60$ \\
CYP2C8 & Gemfibrozil & Strong & $3.1-300$ & $0,15,30,45,60$ \\
CYP2C9 & Fluvoxamine & Weak & $1.6-200$ & $0,5,10,20,30$ \\
CYP2C9 & Miconazole & Moderate & $0.003-10$ & $0,5,10,20,30$ \\
CYP2C9 & Tasisulam & Strong & $0.03-100$ & $0,5,10,20,30$ \\
CYP2C19 & Omeprazole & Weak & $0.01-30$ & $0,15,30,45,60$ \\
CYP2C19 & Fluvoxamine & Strong & $0.03-100$ & $0,5,10,20,30$ \\
CYP2C19 & Fluconazole & Strong & $0.01-30$ & $0,5,10,15,20$ \\
CYP2C19 & Ticlopidine & Strong & $0.1-300$ & $0,15,30,45,60$ \\
CYP2C19 & Osilodrostat & Moderate & $0.1-300$ & $0,15,30,45,60$ \\
CYP2C19 & Moclobemide & Moderate & $3.1-300$ & $0,15,30,45,60$ \\
CYP2D6 & Dronedarone & Weak & $0.0046-10$ & $0,15,30,45,60$ \\
CYP2D6 & Mirabegron & Moderate & $0.0091-20$ & $0,15,30,45,60$ \\
CYP2D6 & Paroxetine & Strong & & $0,15,30,45,60$ \\
\hline
\end{tabular}

Weak $=$ AUCR $(\geq 1.2$ - to $<2.0$-fold $)$, Moderate $=$ AUCR $(\geq 2.0$ - to $<5.0$-fold $)$, Strong $=$ AUCR $(\geq 5.0$-fold $)$. 
TABLE 3

Parameter estimates from pooled human hepatocyte Incubations

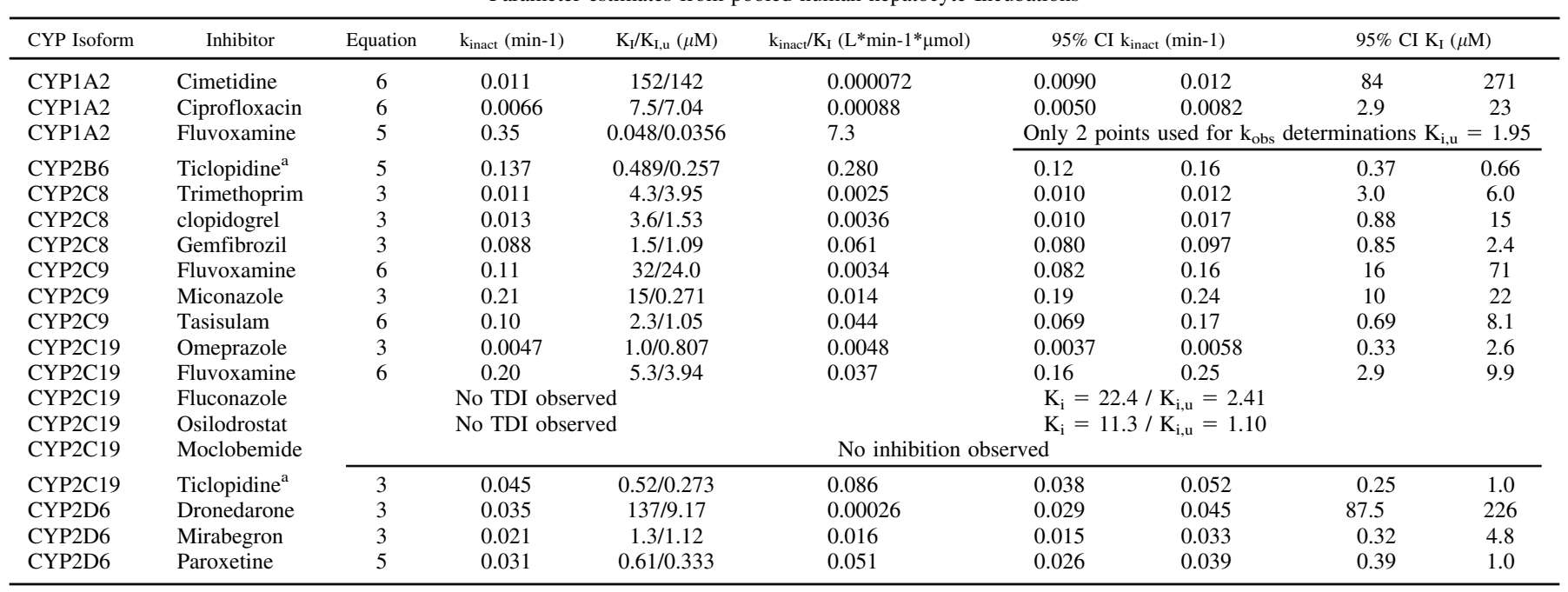

${ }^{\text {a }}$ Ticlopidine parameters were derived using a different pool of hepatocyte donors.

were determined by liquid chromatography tandem mass spectrometry (LC-MS/ MS) analysis using previously validated analytical methods (Perloff et al., 2009).

Linearity of metabolite formation with time was confirmed and Michaelis constant $\left(\mathrm{K}_{\mathrm{m}}\right)$ values determined for all substrates prior to inhibition experiments. Substrate concentrations $\geq$ threefold the Michaelis constant $\left(\mathrm{K}_{\mathrm{m}}\right)$ were chosen for all isoforms. Inhibitors (Table 2) were identified to represent weak, moderate, and strong clinical outcome and incubation concentrations were selected based on available clinical data (reference Supplemental Table 9). Initial preincubation times of $0,15,30,45$, and 60 minutes were used for all inhibitors. Follow-up assays using shorter preincubation times were performed when saturation of inactivation resulted in $<3$ data points available for first order rate constant for inactivation estimated from the slope of residual activity ( $\mathrm{ln}$ ) versus preincubation time at each inhibitor concentration $\left(\mathrm{min}^{-1}\right)\left(\mathrm{k}_{\mathrm{obs}}\right)$ determination.

Cell viability was assessed by trypan blue exclusion for the highest concentration of inhibitor and vehicle control for the longest preincubation time points. Cell suspension aliquots $(25 \mu \mathrm{l})$ were sampled and gently mixed with an equal volume of trypan blue $(0.4 \%)$. Cell number and viability were determined.

Cell viability and observed CYP metabolic activity confirmed a properly functioning hepatocyte model in each assay. Effects of known TDI inhibitors were demonstrated at least once (furafylline (CYP1A2), gemfibrozil (CYP2C8), tienilic acid (CYP2C9), fluvoxamine (CYP2C19), and paroxetine (CYP2D6) but not included in each assay.

TABLE 4

Summary of clinical inhibition data

\begin{tabular}{clcc}
\hline CYP Isoform & Inhibitor & \# of Trials & \# of Substrates \\
\hline \multirow{2}{*}{ 1A2 } & Cimetidine & 7 & 2 \\
& Ciprofloxacin & 11 & 3 \\
& Fluvoxamine & 9 & 6 \\
2 C 8 & Trimethoprim & 6 & 3 \\
& Clopidogrel & 6 & 3 \\
& Gemfibrozil & 30 & 4 \\
2 C9 & Fluvoxamine & 1 & 1 \\
& Miconazole & 1 & 1 \\
& Tasisulam & 1 & 1 \\
2 C19 & Omeprazole & 4 & 2 \\
& Osilodrostat & 1 & 1 \\
& Fluconazole & 6 & 3 \\
& Fluvoxamine & 16 & 5 \\
2D6 & Ticlopidine & 3 & 1 \\
& Dronedarone & 3 & 1 \\
& Mirabegron & 3 & 3 \\
& Paroxetine & 10 & 6 \\
\hline
\end{tabular}

Human liver microsome incubations. Pooled human liver microsomes supplied by XenoTech (H2610 lot\#1710084) were used at a final primary incubation concentration of $1 \mathrm{mg} / \mathrm{mL}$. The primary incubation was equilibrated in a $37^{\circ} \mathrm{C}$ incubator for 10 minutes followed by the initiation of the reaction by addition of NADPH (final concentration $2 \mathrm{mM}$ ). At $0,5,10$, 20 , and 30 minutes preincubation with model inactivator, $7.5 \mu \mathrm{L}$ aliquots were transferred to a plate containing $142.5 \mu \mathrm{L}$ saturating concentrations of probe substrate. Reactions were stopped with $300 \mu \mathrm{L}$ of acetonitrile containing internal standard at 8 minutes for all CYP substrates except paclitaxel (CYP2C8), which was stopped at 12 minutes. Samples were analyzed as previously described (Nishihara et al., 2021).

Positive control inhibitors included furafylline (CYP1A2), gemfibrozil glucuronide (CYP2C8), tienilic acid (CYP2C9), ticlopidine (CYP2C19), and paroxetine (CYP2D6).

LC-MS/MS analysis. Probe substrate metabolites were quantified by LC-MS/ MS analysis as described previously (Perloff et al., 2009; Nishihara et al., 2021).

Calculations. For each assay, metabolite concentrations in the incubated samples were quantified using LC-MS/MS analysis by interpolating from the regression line of the standard curves. Standard curves were produced from least squares linear regression analysis of the ratio of metabolite peak area to internal standard peak area versus concentrations of metabolite.

For each concentration of test compound, the raw data from LC-MS/MS quantitation at each time point were normalized to the corresponding solvent control (no inhibitor) to determine \% CYP activity remaining as shown in Eq. 1. The normalized data were transformed to natural $\log (\ln )$ $\%$ CYP activity remaining and plotted versus the primary incubation time. The slope was determined from the linear portion of the ln \% CYP activity remaining versus primary incubation time curve by linear regression analysis. The negative value of the slope represents $k_{o b s}$, the observed rate constant for inactivation at a specified concentration of inactivator.

$$
\text { CYP activity remaining at }[I]_{t}=\mathrm{C}_{I} / C_{\text {solvent }} * 100
$$

Where $C_{I}$ is the concentration of metabolite formed in the secondary incubation for each concentration of inhibitor at a primary incubation time point and $\mathrm{C}_{\text {solvent }}$ is the concentration of metabolite formed in the secondary incubation for the corresponding solvent control primary incubation time point.

An alternate method to determine the $\mathrm{k}_{\mathrm{obs}}$ was used where \% CYP activity remaining at each concentration of inhibitor at each time point was normalized by the CYP activity in the 0 minute vehicle control (Eq. 2). The ln \% CYP activity remaining was plotted versus primary incubation time. This method resulted in a $\mathrm{k}_{\mathrm{obs}}$ value for the solvent control, first order rate 
TABLE 5

Enzyme degradation rate

\begin{tabular}{lcc}
\hline CYP Isoform & $\mathrm{K}_{\mathrm{deg}}\left(\mathrm{min}^{-1}\right)$ & Reference \\
\hline CYP1A2 & 0.00030 & (Faber and Fuhr, 2004) \\
CYP2B6 & 0.00036 & (Renwick et al., 2000) \\
CYP2C8 & 0.00053 & (Backman et al., 2009) \\
CYP2C9 & 0.00011 & (Renwick et al., 2000) \\
CYP2C19 & 0.00044 & (Liston et al., 2002; \\
CYP2D6 & 0.00023 & Venkatakrishnan and \\
& & Obach, 2005) \\
\hline
\end{tabular}

constant for inactivation estimated from the slope of residual activity (ln) versus preincubation time for the solvent control $\left[\mathrm{min}^{-1}\right.$ ] (may be constrained to equal the $\mathrm{k}_{\mathrm{obs}}$ for the vehicle control) $\left(\mathrm{k}_{\text {solvent }}\right)$, which is a measure of nonspecific loss of activity during incubation.

\% CYP activity remaining at $[I]_{t}=C_{I, t \text { min }} / C_{I, 0 \text { minute solvent }} * 100$

Where $\mathrm{C}_{\mathrm{I}, \mathrm{t}}$ min is the concentration of metabolite formed in the secondary incubation at each inhibitor concentration for each time point and $\mathrm{C}_{\mathrm{I}, 0}$ minute solvent is the concentration of metabolite formed in the secondary incubation in the 0 minute vehicle control.

Non-linear regression models to derive inhibition kinetic parameters. For this study, clinically relevant inactivators of CYPs $1 \mathrm{~A} 2,2 \mathrm{C} 8,2 \mathrm{C} 9,2 \mathrm{C} 19$, and 2D6 were used to assess suspended hepatocytes as a DDI prediction model. The characteristics of CYP inactivators and the determination of $\mathrm{k}_{\text {inact }}$ and $\mathrm{K}_{\mathrm{I}}$ is extensively described in several papers (Orr et al., 2012; Nagar et al., 2014b; Leow and Chan, 2019) and will be minimally addressed here.

Since the inactivator is considered a substrate of the enzyme being inactivated, the Michaelis-Menten (MM) model was used to determine $k_{\text {inact }}$ and $K_{I}$ as shown in Eq. 3 and an adjusted version of the MM model shown in Eq. 6.

If the $\mathrm{k}_{\mathrm{obs}}$ was determined using Eq. 1 for \% CYP activity remaining, then the MM model described in Eq. 3 was used. If the $\mathrm{k}_{\mathrm{obs}}$ was determined using Eq. 2 for \% CYP activity remaining, then the adjusted MM model described in Eq. 6, which includes an extra parameter, $\mathrm{k}_{\text {solvent }}$ for the nonspecific loss of activity in the solvent control. The parameters $k_{\text {inact }}$ and $K_{I}$ were then determined by plotting $\mathrm{k}_{\mathrm{obs}}$ versus [I] and applying nonlinear regression analysis with GraphPad Prism (v. 8, GraphPad Software LLC).

For some experiments nonhyperbolic or atypical MM kinetics such as biphasic and substrate inhibition was observed and Eq. 4 (biphasic) or Eq. 5 (substrate inhibition) models were used. These types of atypical kinetics are considered an artifact of the in vitro system and are discussed in detail elsewhere (Nagar et al., 2014a). Data points to determine $\mathrm{k}_{\text {obs }}$ values were chosen using the linear portion of the curves. The best fit models for $\mathrm{k}_{\text {inact }}$ and $\mathrm{K}_{\mathrm{I}}$ determination were chosen using Akaike Information Criterion value and evaluation of the $95 \%$ confidence intervals for the parameter estimates.

$$
\begin{aligned}
& k_{\mathrm{obs}}=\frac{k_{\text {inact }} \cdot[I]}{K_{I}+[I]} \\
& k_{\mathrm{obs}}=\frac{k_{\text {inact }} \cdot[I]+k_{\mathrm{ratio}} \cdot[I] \cdot[I]}{K_{I}+[I]} \\
& k_{\mathrm{obs}}=\frac{k_{\text {inact }} \cdot[I]}{K_{I}+[I] \cdot\left(1+\frac{[l]}{K_{i}}\right)} \frac{k_{\text {inact }} \cdot[I]}{K_{I}+[I] \cdot\left(1+\frac{[I]}{K_{i}}\right)} \\
& k_{\mathrm{obs}}=k_{\mathrm{solvent}}+\frac{k_{\text {inact }} \cdot[I]}{K_{I}+[I]}
\end{aligned}
$$

Where:

$\mathrm{k}_{\text {inact }}$ is the maximal inactivation rate constant;

$\mathrm{k}_{\mathrm{obs}}$ is the observed rate constant for inactivation;

$\mathrm{K}_{\mathrm{I}}$ is the concentration of inactivator at which the rate constant of inactivation is half maximal;

$\mathrm{k}_{\text {solvent }}$ is the observed rate constant for non-specific loss of activity without inhibitor

$\mathrm{k}_{\text {ratio }}$ is the $\mathrm{k}_{\text {inact }}$ /inhibition constant for reversible inhibition $\left(\mathrm{K}_{\mathrm{i}}\right)$ ratio for the second inactivation site that does not reach saturation; and

$[\mathrm{I}]$ is the concentration of inactivator in the primary incubation.

For some datasets, the $\mathrm{k}_{\text {solvent }}$ parameter was added as a constant by adding the absolute value of the slope for the solvent control to the model. This reduced the number of parameters and therefore the degrees of freedom to achieve a better fit.

Evaluation of in vitro to in vivo extrapolation. Basic models in the regulatory guidance documents were used for the initial analysis (Supplementary Table 5). Equation 7 is the equation recommended in the FDA and PMDA DDI guidelines and incorporates a 50-fold correction factor to the unbound $\mathrm{C}_{\text {max,ss }}$ value. The $\mathrm{R}_{2}$ equation is presented in Eq. 8 and incorporates the enzyme specific rate of degradation $\left(\mathrm{k}_{\mathrm{deg}}\right)$, (reference Table 5). In addition, the evaluation of IVIVE without the correction factor (Eq. 9) was considered by using alternative correction factors such as $3,5,10$, and 15.

$$
\begin{gathered}
k_{o b s}=\frac{k_{\text {inact }} \times 50 \times C_{\max , u}}{K_{I, u}+50 \times C_{\max , u}} \\
R_{2}=\frac{k_{\text {obs }}+k_{\text {deg }}}{k_{\text {deg }}} \frac{k_{\text {obs }}+k_{\text {deg }}}{k_{\text {deg }}} \geq 1.25
\end{gathered}
$$

TABLE 6

Input parameters for the mechanistic static model

\begin{tabular}{lcccccccl}
\hline Inhibitor & Molecular Weight g/Mol & Log P or D & $\mathrm{F}_{\mathrm{u}, \mathrm{p}}$ & $\mathrm{F}_{\mathrm{a}}$ & $\mathrm{F}_{\mathrm{g}}$ & $\mathrm{K}_{\mathrm{a}} \mathrm{min}^{-1}$ & $\mathrm{R}_{\mathrm{b}}$ & \multicolumn{1}{c}{ References } \\
\hline Cimetidine & 252.34 & 0.48 & 0.81 & 1 & 0.92 & 0.012 & 0.97 & (Varma et al., 2010; Burt et al., 2016) \\
Ciprofloxacin & 331.346 & 0.3 & 0.60 & 0.75 & 0.98 & 0.01 & 0.75 & (Varma et al., 2010) \\
Fluvoxamine & 318.337 & 3.0 & 0.23 & 1 & 0.5 & 0.012 & 1.5 & (Jogiraju et al., 2021) \\
Trimethoprim & 290.321 & 0.91 & 0.50 & 1 & 0.8 & 0.0082 & 1 & (Kim et al., 2016) \\
Clopidogrel & 321.826 & 2.58 & 0.02 & 0.5 & 1 & 0.08 & 0.57 & (Xu et al., 2020) \\
Clopidogrel glucuronide & 483.92 & 2.58 & 0.1 & $\mathrm{NA}$ & $\mathrm{NA}$ & $\mathrm{NA}$ & 0.57 & (Tornio et al., 2014) \\
Gemfibrozil & 250.336 & 4.3 & 0.03 & 1 & 1 & 0.1 & 0.825 & (Varma et al., 2015) \\
Gemfibrozil glucuronide & 426.5 & 3.3 & 0.115 & $\mathrm{NA}$ & $\mathrm{NA}$ & $\mathrm{NA}$ & 0.825 & \\
Miconazole & 416.134 & 5.96 & 0.08 & 0.1 & 0.01 & 0.03 & 1.5 & (O'Reilly et al., 1992; Miki et al., 2011) \\
Tasisulam & 437.09 & 3.8 & 0.01 & $\mathrm{NA}$ & $\mathrm{NA}$ & $\mathrm{NA}$ & $\mathrm{NA}$ & (Perkins et al., 2018) \\
Omeprazole & 345.42 & 2.43 & 0.05 & 1 & 1 & 0.1 & 1 & (Marsousi et al., 2018) \\
Osilodrostat & 227.241 & 2.11 & 0.636 & 1 & 1 & 0.0467 & 0.85 & (Armani et al., 2017) \\
Fluconazole & 306.275 & 0.2 & 0.89 & 0.98 & 1 & 0.0292 & 1 & (Marsousi et al., 2018) \\
Ticlopidine & 263.786 & 3.6 & 0.02 & 1 & 0.5 & 0.03 & 0.55 & Default values used \\
Dronedarone & 556.764 & 5.28 & 0.01 & 1 & 0.898 & 0.0136 & 1 & (Djebli et al., 2015) \\
Mirabegron & 396.513 & 2.1 & 0.27 & 1 & 0.68 & 0.00617 & 1.42 & (Konishi et al., 2019) \\
Paroxetine & 329.369 & 3.55 & 0.05 & 0.93 & 1 & 0.017 & 1.26 & (Marsousi et al., 2018) \\
\hline
\end{tabular}




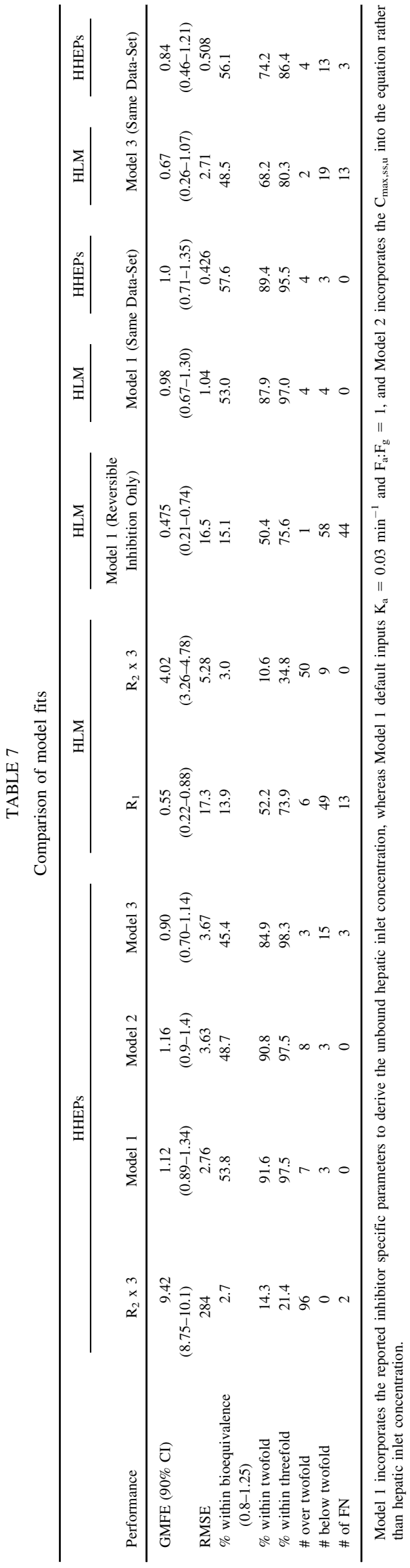

$$
k_{\text {obs }}=\frac{k_{\text {inact }} \times C_{\max , u}}{K_{I, u} \times C_{\max , u}}
$$

To refine the quantitative prediction, the mechanistic static model (MSM), which enables additive perpetrators, reported elsewhere (Fahmi et al., 2008; Isoherranen et al., 2012) was also used (Eq. 10).

$$
\begin{gathered}
\frac{A U C_{i}}{A U C}=\frac{1}{F_{g}+\left(1-F_{g}\right) \times\left(\sum_{k=1}^{n} \frac{f m(E)_{g, k}}{A_{g, k} \times B_{g, k} \times C_{g, k}}+1-\sum_{k=1}^{n} f m(E)_{g, k}\right)} \\
x \cdot \frac{1}{\sum_{k=1}^{n} \frac{f m(E)_{h, k}}{A_{h, k} \times B_{h, k} \times C_{h, k}}+\left(1-\sum_{k=1}^{n} f m(E)_{h, k}\right)}
\end{gathered}
$$

Where:

$\mathrm{A}=$ reversible inhibition, $\mathrm{B}=$ time-dependent inhibition, $\mathrm{C}=$ induction, $\mathrm{g}=$ gut, $\mathrm{h}=$ liver and $\mathrm{k}=$ enzyme.

Since the enzymes used in this analysis are minimally expressed in enterocytes (Paine et al., 2006; Thelen and Dressman, 2009; Xie et al., 2016), or their expression does not impact DDI outcome (CYP2C9/ CYP2C19), (see result section: verification of the lack of importance of CYP2C intestinal expression to DDI) the gut component was removed from Eq. 10. Additionally, since the inhibitors that were evaluated were not inducers, the induction terms were likewise removed, resulting in Eq. 11, which includes Eq. 12, representing the reversible inhibition, and Eq. 13, the time-dependent inhibition in liver.

$$
\begin{gathered}
\frac{A U C_{i}}{A U C}=\frac{1}{\sum_{k=1}^{n} \frac{f m(E)_{h, k}}{A_{h, k} \times B_{h, k}}+\left(1-\sum_{k=1}^{n} f m(E)_{h, k}\right)} \\
A_{h, k}=1+\frac{I_{\text {inlet }, \text { max }, u}}{K_{i, u}} \\
B_{h, k}=1+\frac{k_{\text {inact }} \times I_{\text {inlet }, \text { max }, u}}{k_{\text {deg }, h} \times\left(I_{\text {inlet }, \text { max }, u}+K_{I, u}\right)}
\end{gathered}
$$

Calculation of maximal hepatic inlet concentration was conducted using Eq. 14, and the unbound hepatic inlet concentration $\left(\mathrm{I}_{\text {inlet,max,u) }}\right.$ was calculated with Eq. 15.

$$
\begin{gathered}
I_{\text {inlet, } \max }=R b \times C_{\text {max }, \text { plasma }}+\frac{F a \times F g \times K a \times \text { Dose }}{Q h} \\
I_{\text {inlet }, \text { max }, u}=\frac{(1-H) \times f_{u, p}}{R b} \times I_{\text {inlet, } \text { max }}
\end{gathered}
$$

Where $\mathrm{H}$ is the hematocrit and assumed to be 0.45 and $\mathrm{Rb}$ is the blood-toplasma ratio.

Multiple iterations of the above model with various $[I]$ input values were considered including:

Model 1: Using unbound hepatic inlet concentration as described in regulatory guidance;

Model 2: Using unbound hepatic inlet concentration calculated with default values; and

Model 3: Inputting unbound systemic $\mathrm{C}_{\text {max,ss }}$ in place of $\mathrm{I}_{\text {inlet,max,u}}$.

All models were evaluated considering the range of published fraction metabolized through the pathway (Fm) values (Supplemental Table 3), and Model 1 used published $\mathrm{Ka}, \mathrm{Fa}$ and $\mathrm{Fg}$ values where available (Table 6), default values used for Model 2 were Ka (0.03 minute-1), $\mathrm{Fa}: \mathrm{Fg}=1$ and $\mathrm{Rb}=0.55$. The best universally fitting Fm was selected for the optimized data and is depicted in bold in Supplemental Table 3.

In silico estimation of unbound $K_{i}$ and $K_{I}$ values. The Kilford equation (Kilford et al., 2008), Eq. 16, was used to estimate the unbound inhibition parameters. In the case of the experimental conditions employed in these studies, the hepatocyte concentration was 50,000 cells $(1 \times$ $10^{6}$ cells $/ \mathrm{mL}$ ), and there was no additional protein present in the media. An intracellular volume of $6.48 \mathrm{pL}$ was used; the incubation volume was $50 \mu \mathrm{L}$, resulting in a $\mathrm{V}_{\mathrm{R}}$ of $0.00648\left(\right.$ Note $\mathrm{V}_{\mathrm{R}}=\mathrm{V}_{\text {cell }} \mathrm{x}$ $\left.\mathrm{V}_{\text {inc }}\right)$. $\log \mathrm{P}$ or $\mathrm{D}$ values reported in the literature for the inhibitors were used (Table 6). 
A

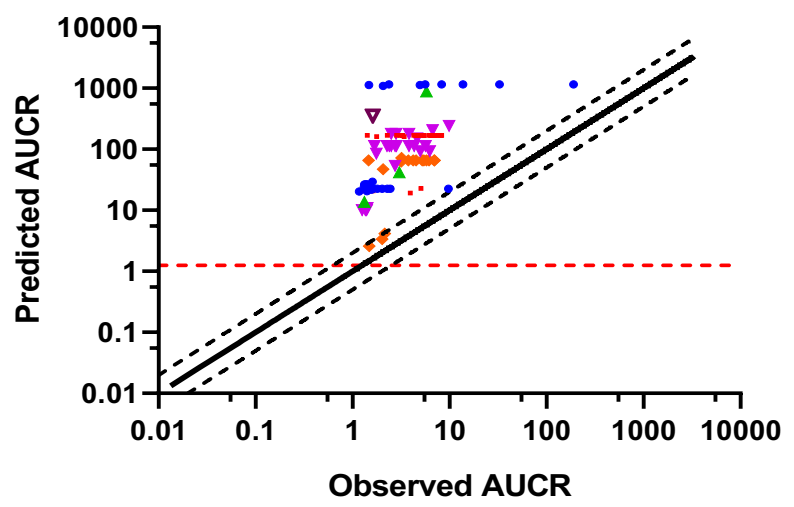

C

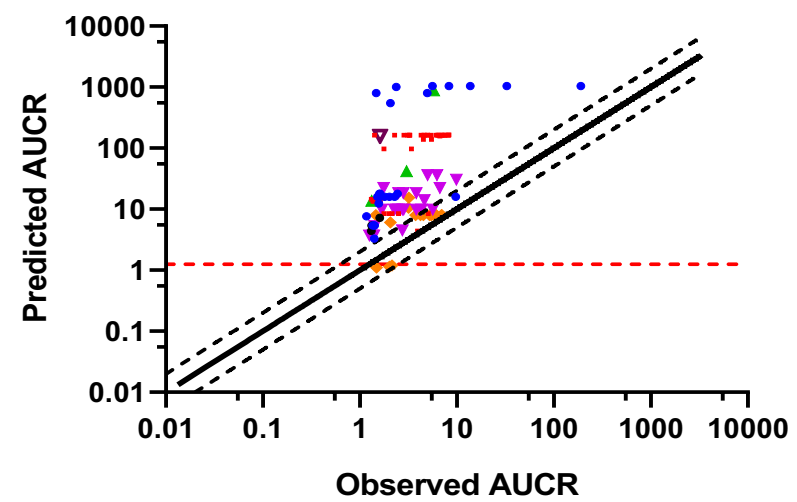

B

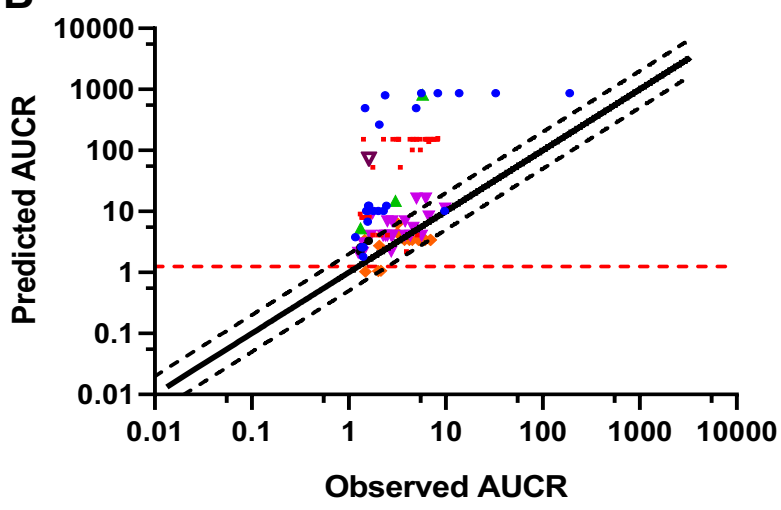

- CYP1A2

- CYP2C8

- CYP2C9

- CYP2C19

- CYP2D6

จ CYP2B6

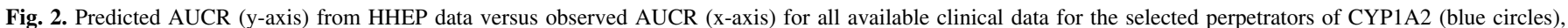

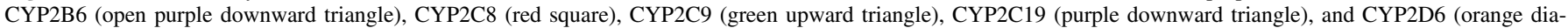

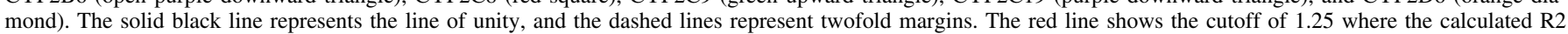

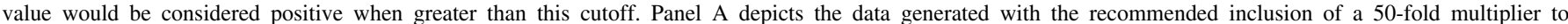

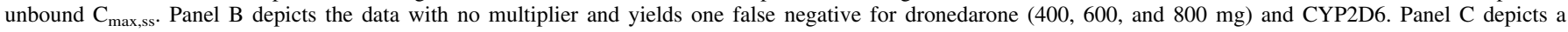
threefold multiplier, which reduces false negatives to 0 .

$$
f_{u, \text { hep }}=\frac{1}{1+125 \times V_{R} \times 10^{0.072 \times \log P \text { or } D+0.067 \times \log P \text { or } D-1.126}}
$$

Correction of in vitro derived $\mathrm{IC}_{50}$ values based on saturating substrate concentrations used in the time-dependent inhibition assay was conducted according to Eq. 17 (Cheng and Prusoff, 1973) and assuming competitive inhibition.

$$
K_{i}=\frac{I C_{50}}{1+\frac{[\text { Substrate }]}{K_{m}}}
$$

Statistical analysis of the goodness of fit for IVIVE models evaluated. The accuracy of the prediction of the individual models was evaluated by deriving the geometric mean fold error (GMFE) according to Eq. 18 and the root mean square error (RMSE) according to Eq. 19. GMFE closest to 1 represents the best fit, whereas RMSE approaching 0 does.

$$
\begin{gathered}
G M F E=10^{\text {mean }}\left(\left|\log \frac{\text { predicted } D D I}{\text { observed DDI }}\right|\right) \\
R M S E=\sqrt{\frac{\sum(\text { predicted } D D I-\text { observed } D D I)^{2}}{\text { number of predictions }}}
\end{gathered}
$$

\section{Results}

Selection of Clinically Relevant Inhibitors for In Vitro Data Generation

The search of the UW-DIDB identified weak, moderate, and strong inhibitors toward CYPs 1A2, 2C8, 2C9, 2C19, and 2D6 (Table 2). Although an attempt was made to identify clinically relevant inhibitors of CYP2B6, studies were limited to no effect or weak inhibition, thus in vitro evaluation was not further pursued and IVIVE was conducted using reported and/or historical values (Supplementary Table 4). Clinical data used for the IVIVE evaluation of selected inhibitors of CYPs 1A2, 2C8, 2C9, 2C19, and 2D6 is presented in Supplementary Table 9.

\section{TDI Results}

Time-dependent inhibition was observed for 16 of the 19 evaluated inhibitors, and kinetic parameters could be confidently determined for them using hepatocytes (Table 3). Mild TDI was observed for cimetidine in HLM; however, inactivation parameters could not be confidently estimated. TDI was not observed in HLM, under the experimental conditions, for any other clinically weak inhibitors, but was observed for one moderate and three strong inhibitors. The positive control inhibitors used in the HLM assay demonstrated expected and robust response with kinetic parameters in line with those reported previously. In human hepatocyte incubations, the $\mathrm{k}_{\text {inac }} / \mathrm{K}_{\mathrm{I}}$ ratios trended with the classification from weak to strong clinical inhibition such that the lower the ratio the weaker the observed clinical effect.

Time-dependent inhibition of CYP1A2 in hepatocytes was observed for cimetidine, ciprofloxacin, and fluvoxamine, using phenacetin as the probe substrate. Fluvoxamine showed potent and rapid inhibition of enzyme activity after a 5-minute preincubation but did not show a further decrease in activity with increasing preincubation time. As a result, 


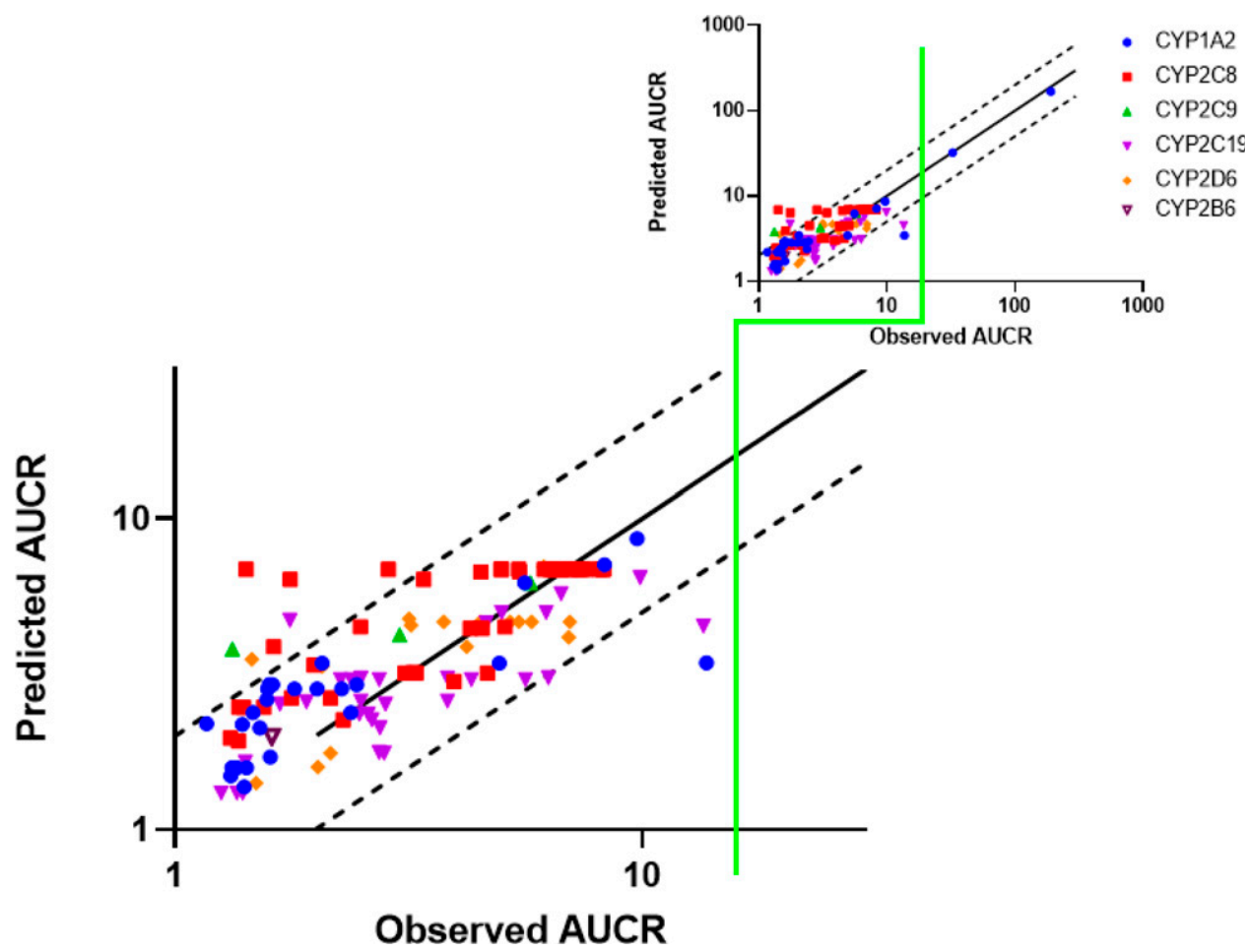

Fig. 3. Predicted AUCR (y-axis) from HHEP data versus observed AUCR (x-axis) for all available clinical data for the selected perpetrators of CYP1A2 (blue circles), CYP2C8 (red square), CYP2C9 (green upward triangle), CYP2C19 (purple downward triangle), CYP2D6 (orange diamond), and CYP2B6 (open purple downward triangle). The solid black line represents the line of unity, and the dashed lines represent twofold margins. The predicted AUCR was generated using the mechanistic static model with the inhibitor specific parameters for $F_{a}, F_{g}, K_{a}$, and $R_{b}$ (Table 6) and the $F_{m}$ value indicated in bold in Supplemental Table 3. The inlet graph expands the axis to include the strong inhibition observed for fluvoxamine against CYP1A2 substrates.

only 2 data points were available to estimate $\mathrm{k}_{\mathrm{obs}}$ values (Supplemental Fig. 1) resulting in a potential underestimation of inhibitory potency. TDI of CYP1A2 was observed in HLM for only the strong inhibitors, fluvoxamine and furafylline, and not for the weak and moderate inhibitors. The kinetic parameters for fluvoxamine were $\mathrm{K}_{\mathrm{I}}$ of $1.81 \mu \mathrm{M}$ and a $\mathrm{K}_{\text {inact }}$ of 0.0747 minute $^{-1}$ and for furafylline were $\mathrm{K}_{\mathrm{I}}$ of $22.5 \mu \mathrm{M}$ and a $\mathrm{K}_{\text {inact }}$ of 0.372 minute $^{-1}$.

Time-dependent inhibition of CYP2C8 in hepatocytes was observed for trimethoprim, clopidogrel, and gemfibrozil, using amodiaquine as the probe substrate. The inhibition parameters determined from clopidogrel and gemfibrozil were likely due to the glucuronide metabolites as described elsewhere (Ogilvie et al., 2006; Tornio et al., 2014). TDI of CYP2C8 was not observed with the test set in HLM although the positive control, gemfibrozil glucuronide, yielded a total $\mathrm{K}_{\mathrm{I}}$ of $29.8 \mu \mathrm{M}$ and a $\mathrm{K}_{\text {inact }}$ of 0.04 minute $^{-1}$.

Time-dependent inhibition of CYP2C9 in hepatocytes was observed for fluvoxamine, miconazole, and tasisulam, using diclofenac as the probe substrate. For fluvoxamine, substantial cytotoxicity was observed at concentrations of $100 \mu \mathrm{M}$ and above (trypan blue viability of $33 \%$ at 200 $\mu \mathrm{M}$ with a 30-minute preincubation). As it is unclear what, if any, impact the decreased viability might have on CYP enzyme activity, the 200 and $300 \mu \mathrm{M}$ data points were excluded from analysis. TDI of CYP2C9 was not observed with the test set in HLM although the positive control, tienilic acid, yielded a total $\mathrm{K}_{\mathrm{I}}$ of $4.35 \mu \mathrm{M}$ and a $\mathrm{K}_{\text {inact }}$ of 0.108 minute ${ }^{-1}$.

Time-dependent inhibition of CYP2C19 was evaluated in human hepatocytes for omeprazole, fluvoxamine, fluconazole, osilodrostat, moclobemide, and ticlopidine. TDI was observed and $\mathrm{K}_{\mathrm{I}}$ and $\mathrm{k}_{\text {inact }}$ values were determined for omeprazole and fluvoxamine. Fluconazole and osilodrostat did not demonstrate TDI, but as both compounds resulted in comparable concentration dependent inhibition at all preincubation times, $\mathrm{K}_{\mathrm{i}}$ values were estimated from the $\mathrm{IC}_{50}$ determined at the first time point according to Eq. 17. Moclobemide did not demonstrate any inhibition of CYP2C19 activity in hepatocytes despite resulting in a clinically moderate inhibition of omeprazole clearance [area under the concentration curve ratio (AUCR) $=2.07]$. TDI was not observed in HLM for omeprazole, osilodrostat, or fluvoxamine under the incubation conditions used. There was TDI observed for ticlopidine with a total $\mathrm{K}_{\mathrm{I}}$ of $85.7 \mu \mathrm{M}$ and a $\mathrm{K}_{\text {inact }}$ of 0.111 minute $^{-1}$.

The inhibition potential for ticlopidine was investigated in this pooled lot of human hepatocytes but did not demonstrate TDI. Of note, historical studies using an alternate set of three donors of hepatocytes have demonstrated time-dependent inhibition of CYP2C19 by ticlopidine and kinetic parameters from those studies were used for the clinical risk assessment. The reason for the difference between donors is unclear. In pooling donors, any impact of polymorphic enzymes should be reduced (Ramsden et al., 2009); however, genotyping data for the donors used in these studies was not available.

Time-dependent inhibition of CYP2D6 was observed for dronedarone in hepatocytes only and for mirabegron and paroxetine in both HLM and human hepatocytes.

Resulting graphs depicting the ln \% remaining CYP activity versus incubation time and $\mathrm{k}_{\mathrm{obs}}$ versus inhibitor concentration are provided in Supplemental Fig. 1.

\section{Verification of the Lack of Importance of CYP2C Intestinal Expression to DDI}

It is well recognized that intestinal CYP3A contributes significantly to observed DDI after oral administration of CYP3A perpetrators (Ramsden et al., 2019; Yamada et al., 2020). The impact of intestinal expression of other CYP enzymes is less clear. It is reported that 


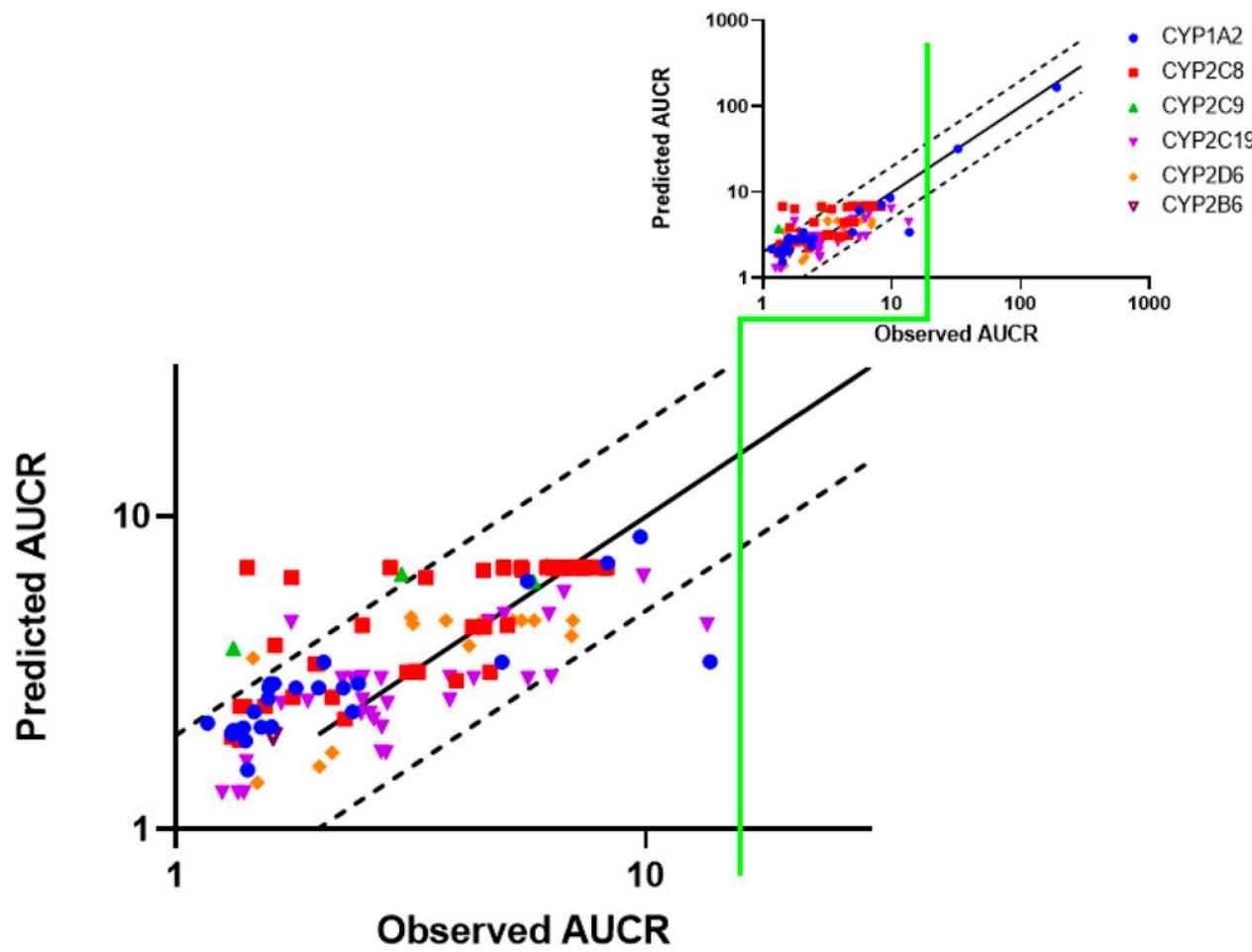

Fig. 4. Predicted AUCR (y-axis) from HHEP data versus observed AUCR (x-axis) for all available clinical data for the selected perpetrators of CYP1A2 (blue circles), CYP2C8 (red square), CYP2C9 (green upward triangle), CYP2C19 (purple downward triangle), CYP2D6 (orange diamond), and CYP2B6 (open purple downward triangle). The solid black line represents the line of unity, and the dashed lines represent twofold margins. The predicted AUCR was generated using the mechanistic static model with the default parameters for $\mathrm{F}_{\mathrm{a}}(1), \mathrm{F}_{\mathrm{g}}(1), \mathrm{K}_{\mathrm{a}}\left(0.03\right.$ minute $\left.{ }^{-1}\right)$, and $\mathrm{R}_{\mathrm{b}}(0.55)$ and the $\mathrm{F}_{\mathrm{m}}$ value indicated in bold in Supplemental Table 3. The inlet graph expands the axis to include the strong inhibition observed for fluvoxamine against CYP1A2 substrates.

CYP2C9 is the next most abundantly expressed CYP representing $14 \%$ of the detected intestinal CYP content, followed by CYP2C19 (2\%) and CYP2D6, whereas neither CYP1A2 or CYP2C8 were detected (Paine et al., 2006; Xie et al., 2016). To evaluate the importance of CYP2C9 intestinal expression toward observed DDIs, the clinical inhibition and induction data were reviewed for inhibitors and inducers evaluated against the substrates when dosed IV (hepatic) and orally (hepatic + intestinal) (Supplemental Table 6). As evidenced by the similar magnitudes of change observed when substrates were dosed IV or orally, after administration of the inhibitor or inducer, the impact of intestinal CYP2C9 toward the observed DDI is limited. In addition, the $\mathrm{F}_{\mathrm{g}}$ reported for common $\mathrm{CYP} 2 \mathrm{C} 9$ substrates including warfarin, tolbutamide, celecoxib, and phenytoin are $>0.9$, whereby the max percent AUC increase from inhibition at the intestinal level is calculated to be $11 \%$. Since the expression of CYP2C19 and CYP2D6 in the gut is much less than CYP2C9, an assumption is made that the impact of intestinal activity on the magnitude of DDI is also likely to be limited.

How Does the Data Generated in This Study Compare with Literature Values Reported in HLM? Although the scope of the enclosed work did not originally include comparative evaluation of TDI for non-CYP3A enzymes using recombinant CYPs or HLM, conducting these studies in human hepatocytes isn't trivial and evaluation of the predictivity of parameters reported for HLM was performed. To facilitate this analysis, all available in vitro parameters for the selected inhibitors were collated from literature (Supplemental Table 7). In some cases, the inhibitor resulted in time-dependent inhibition of multiple CYPs (cimetidine, dronedarone, fluvoxamine, omeprazole, paroxetine, and ticlopidine). Thus, it is important to understand the selectivity of the clinical probe substrate and whether the potential inhibition of other
CYPs involved in its metabolism needs to be considered in the DDI risk assessment. The available literature data for the inhibitors selected in the analysis conducted herein was limited. Published values were available for gemfibrozil, gemfibrozil glucuronide, omeprazole, osilodrostat, paroxetine, and ticlopidine (for both CYP2B6 and CYP2C19). The inhibition parameters were generated in-house for fluvoxamine, gemfibrozil glucuronide, ticlopidine, mirabegron, and paroxetine. The values were corrected to unbound values using the in silico approach reported by Hallifax and Houston, 2006. In addition, an attempt was made to derive the TDI parameters for the selected test set using the standard protocols established within Takeda. Kinetic parameters could only be derived for a limited number of the inhibitors using HLM under the incubation conditions used. A recent publication highlighted the critical role of passive permeability to differences between clearance and inhibition parameters derived from HHEP and HLM (Keefer et al., 2020). To understand whether the time dependency observed in hepatocytes may be an artifact of low/slow permeability followed by direct inhibition, reported information on the biopharmaceutics classification system and direct inhibition parameters was collected (Supplemental Table 8). These data were used to evaluate the potential for reversible inhibition to recover the observed clinical DDI using the MSM. Using only the reversible inhibition parameters resulted in 44 false negative trials and a large underprediction (58/119 over twofold below the observed magnitude) (Table 7). These results suggest that delayed permeability, followed by direct inhibition, cannot explain the lack of TDI observed in HLM for weak and moderate inhibitors. An additional approach would be to experimentally derive the $\mathrm{K}_{\text {puu }}$ values in hepatocytes, rather than relying on in silico values. There are a number of proposed methods to derive this value although no consensus has been 


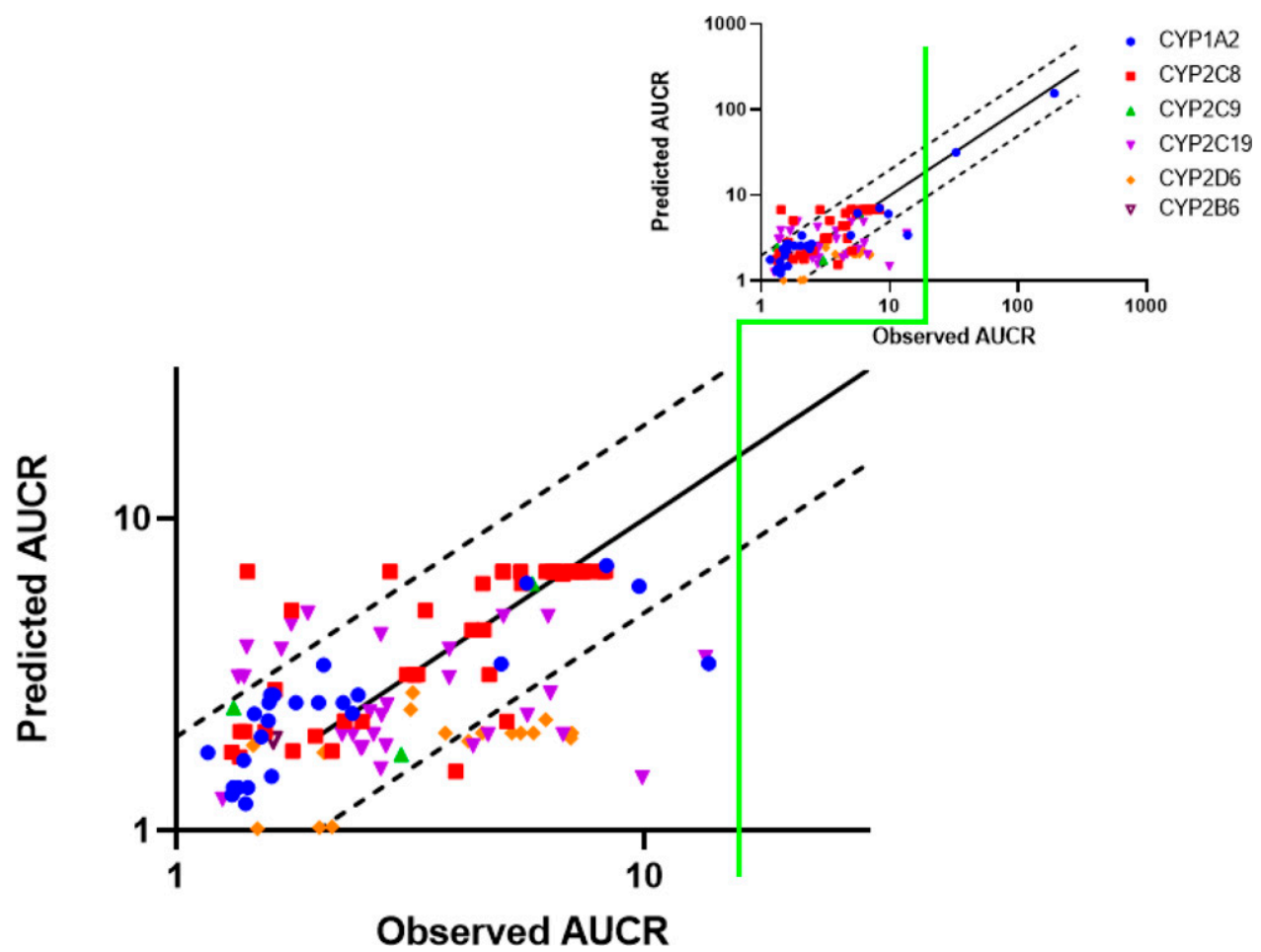

Fig. 5. Predicted AUCR (y-axis) from HHEP data versus observed AUCR (x-axis) for all available clinical data for the selected perpetrators of CYP1A2 (blue circles), CYP2C8 (red square), CYP2C9 (green upward triangle), CYP2C19 (purple downward triangle), CYP2D6 (orange diamond), and CYP2B6 (open purple downward triangle). The solid black line represents the line of unity, and the dashed lines represent twofold margins. The predicted AUCR was generated using the mechanistic static model with the unbound $\mathrm{C}_{\text {max,ss }}$ and the $\mathrm{F}_{\mathrm{m}}$ value indicated in bold in Supplemental Table 3. The inlet graph expands the axis to include the strong inhibition observed for fluvoxamine against CYP1A2 substrates. Of note, this model failed to identify the clinical relevance of dronedarone toward metoprolol, a CYP2D6 substrate.

reached, and therefore $\mathrm{K}_{\text {puu }}$ values were not determined in the enclosed studies (Chu et al., 2013; Mateus et al., 2013; Riccardi et al., 2016).

Clinical Risk Assessment from In Vitro Inhibition Parameters. The clinical risk assessment was conducted following the recommendations set forth in the regulatory documents (FDA, EMA, and PMDA). The first step was to use the basic models that consider the inhibition kinetic parameters and the maximal unbound plasma concentration at steady state $\left(\mathrm{C}_{\text {max,ss,u }}\right)$ but do not incorporate substrate specific parameters (Eq. 7-9). The nominal $\mathrm{K}_{\mathrm{I}}$ values were corrected to unbound $\mathrm{K}_{\mathrm{I}}$ values using the predicted nonspecific binding to hepatocytes or HLM (Eq. 16). The unbound $\mathrm{K}_{\mathrm{I}}$ values were used in the subsequent equations to assess the clinical risk. The degradation rates presented in Table 5 were input into Eq. 8, dependent on the CYP being inhibited. While the inhibitor $\mathrm{C}_{\text {max,ss,u }}$ values for the enclosed dataset are known, it should be appreciated that this value is often based on a prediction using preclinical data prior to when first-in-human or multiple dose clinical studies have been conducted. The impact of the inhibitor concentration input value should therefore be considered during the clinical risk assessment for new chemical entities. The $\mathrm{R}_{2}$ value generated with the basic model was compared with the observed AUCR (Supplementary Table 9). The resulting $R_{2}$ values using Eq. 8 significantly overpredicted the observed magnitude of DDI (Fig. 2A, Table 7) when $\mathrm{K}_{\text {obs }}$ were calculated with 50x unbound $\mathrm{C}_{\max }$ (Eq. 9). When the 50-fold correction factor was removed according to Eq. 7, dronedarone, a weak to moderate inhibitor of CYP2D6, resulted in a false negative at all three clinical dose levels studied (Fig. 2B). The $800 \mathrm{mg}$ dronedarone became a true positive when applying a correction factor of 3 to the $\mathrm{R}_{2}$ equation; however, both the 400 and $600 \mathrm{mg}$ dose level predictions were considered false negatives (Fig. 2C, Table 7). The next step was to evaluate various iterations of the MSM (Eq. 10). The MSM model incorporates both inhibitor and substrate specific parameters. The $F_{m}$ value(s) for each substrate was collected from the literature (Supplemental Table 3). In cases where multiple $\mathrm{F}_{\mathrm{m}}$ values were reported, individual and mean values were evaluated in the prediction. In terms of inhibitor specific parameters, the literature was searched for $K_{a}, F_{a}, F_{g}$, and $R_{b}$ to support estimation of the hepatic inlet concentration (Table 6) using Eqs. 14 and 15. When the reported values were used to estimate the unbound hepatic inlet concentration and the optimal $F_{m}$ values for the substrates were used, there were no false negatives and there was good quantitative prediction observed (Fig. 3, Table 7). In this case, 109 of the 119 (92\%) clinical studies were predicted within twofold of the observed AUCR and 64 were predicted within bioequivalence or between 0.8- to 1.25 -fold of the observed. The magnitude of seven clinical studies was overpredicted ( $>$ twofold predicted/observed) and three were underpredicted $(<0.5$ predicted/observed). Trimethoprim with repaglinide was overpredicted by 2.4 -fold. There were three trials with gemfibrozil, which were overpredicted using repaglinide as the probe substrate for CYP2C8 ranging from 2.4- to 4.8-fold; of note, 20 other similarly designed trials fell within twofold of the observed with 15 of them within bioequivalence. A similar observation was made for ticlopidine with omeprazole where one trial was overpredicted by 2.6 -fold and the other two trials were predicted within bioequivalence. If the average of the clinical results is used rather than discreet AUC values, these are no longer overpredicted. Similarly, the inhibitors that were underpredicted (fluconazole and fluvoxamine) were well-predicted in all of the other clinical studies, $4 / 6$ and $8 / 9$, respectively. The analysis of these trends is presented in Supplemental Fig. 2. Therefore, it is likely that the variability in outcome observed between clinical interaction studies should be considered in the risk assessment. The importance of substrate selectivity in the magnitude of DDI can be highlighted by the magnitude of inhibition observed for fluvoxamine against CYP1A2 substrates, where the predicted AUCR ranges from 2.38-fold with theophylline to 
A

\begin{tabular}{|l|l|l|l|l|l|l|l|}
\hline CYP & Category & \multirow{2}{*}{ Inhibitor } & \multicolumn{2}{|c|}{ HHEP } & \multicolumn{2}{|c|}{ HLM } & \multicolumn{1}{|c|}{ Source } \\
for HLM
\end{tabular}

B

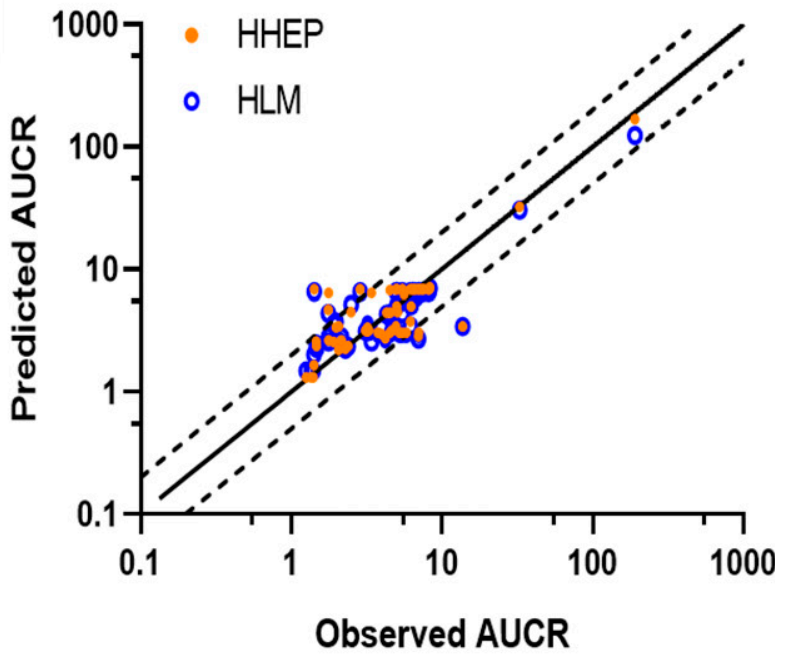

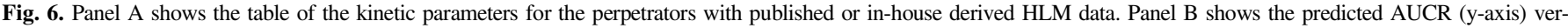

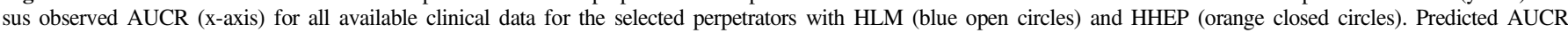
was derived using the unbound hepatic inlet concentration. The solid black line represents the line of unity, and the dashed lines represent twofold margins.

168-fold with ramelteon. Likewise, the $\mathrm{F}_{\mathrm{mCYP} 1 \mathrm{~A} 2}$ for theophylline (0.58) is much lower than that of ramelteon (0.995) as was the magnitude of DDI observed, 1.47- to 2.38-fold for theophylline and 190-fold for ramelteon. Since it is appreciated that the inhibitor specific parameters are often not known during early DDI risk assessment, default values of 1 for $F_{a}: F_{g}, 0.03$ minute $^{-1}$ for $K_{a}$ and 0.55 for $R_{b}$ were also evaluated to derive the unbound hepatic inlet concentration. This also resulted in zero false negatives and 108 of $119(90.8 \%)$ trials within twofold and $58(49 \%)$ within bioequivalence. In the case of missed predictions, most (8/11) were overpredicted (Fig. 4). Lastly, the MSM was evaluated using the $\mathrm{C}_{\text {max,ss,u }}$ rather than the unbound hepatic inlet concentration. Using this inhibitor input value resulted in a higher number of underpredictions $(15<0.5)$ and reduced number of values within twofold (84.9\%). Clinical risk assessment using the MSM with the $\mathrm{C}_{\text {max,ss,u }}$ resulted in dronedarone as a false negative (Fig. 5). Using the full dataset available with the HHEPs data and considering the GMFE closest to 1 and the lowest RMSE, Model 1 performed the best followed by Model 2 (default values to derive the hepatic inlet concentration) and lastly Model 3 (Table 7). Since using the $C_{\text {max,ss,u }}$ resulted in a significant increase in the number of underpredicted DDI outcome $(3 \rightarrow 15)$, evaluation of the average unbound systemic plasma concentration at steady state was not conducted. Considering that the available clinical and in vitro parameter dataset for HLM was significantly smaller than HHEPs (66 versus 119) a direct comparison between them was made (Table 7, two far right columns, Fig. 6). In general, the parameters derived from HHEPs performed slightly better than those from HLM when comparing the GMFE and RMSE values. The quantitative accuracy was also higher in HHEPs than HLMs. Consistent with the data observed for the full HHEPs dataset, there were no false negatives (FN) when using the unbound hepatic inlet concentration; whereas there were 3 and 13, for HHEPs and HLM, respectively when inputting the $\mathrm{C}_{\max , \mathrm{ss}, \mathrm{u}}$. In the case where $\mathrm{C}_{\mathrm{max}, \mathrm{ss}, \mathrm{u}}$ was used as the input parameter, there were 13 false negatives using the HLM data including trials with ticlopidine (3/3), mirabegron (1/3), and paroxetine (9/10) and 3 false negatives using the hepatocyte data (3/4, omeprazole trials), (Table 7). Taken together, these data suggest that parameters generated from either HLM or HHEPs coupled with the MSM using $\mathrm{I}_{\text {inlet,max, }}$ as the input results in quantitative prediction of magnitude of DDI with no false negatives. Since good quantitative predictions were possible using the MSM, which is much easier and more accessible to researchers, Physiologically-Based Pharmacokinetic (PBPK) modeling was not conducted. It is possible that some of the overpredictions might be reduced with PBPK modeling.

\section{Discussion}

Clinically relevant TDI has been reported for multiple CYP enzymes, although clinical risk assessments and IVIVE efforts have historically focused on CYP3A as the primary enzyme responsible for the majority of DDI (Obach et al., 2007; Mao et al., 2011; Kenny et al., 2012; Vieira et al., 2014; Tseng et al., 2021). Given the importance of identifying DDI liabilities during drug development, regulatory agencies have proposed guidance on evaluating the DDI potential for NCEs (EMA, FDA, and PMDA). Although clinically relevant TDI has been reported for non-CYP3A enzymes including CYP1A2, CYP2B6, CYP2C8, CYP2C9, CYP2C19, and CYP2D6, to our knowledge a systematic review has not been conducted, and therefore it is unclear whether the proposed recommendations, based on CYP3A data, are appropriate for characterizing the clinical inhibition risk of these non-CYP3A isoforms.

It is appreciated that traditional in vitro studies tend to overpredict the risk for DDI and can be based on a number of assumptions. Similar observations have been reported for competitive inhibition, whereby the inhibitory parameters associated with compounds likely to accumulate within cells due to active uptake were markedly different in experiments conducted using HLM and human hepatocytes (Brown et al., 2010). To this end, experiments were designed using pooled human hepatocytes to derive TDI kinetic parameters with a focus on non-CYP3A enzymes. To investigate the utility of suspended hepatocytes as a tool to better predict TDI, the literature was mined for clinically relevant weak, moderate, and strong inhibitors of CYPs 1A2, 2C8, 2C9, 2C19, and 2D6 using the 
UW-DIDB. Inhibitors were selected with clinical inhibition observed under steady state conditions. The literature was further searched to evaluate whether in vitro induction parameters were available for the inhibitor test set, and where these parameters were not available, the assumption was made that the inhibitor was not an inducer. Furthermore, whether the inhibitors were substrates or inhibitors of major drug transporters was also considered. In vitro incubation conditions were established based on validation work conducted by Corning Life Sciences. The in vitro test concentrations used in the evaluation were determined considering the clinical concentrations, solubility, existing data, and toxicity potential with the goal to enable estimation of the in vitro kinetic parameters. The time points were selected to ensure adequate sensitivity for deriving the inhibition rate constants $\mathrm{k}_{\mathrm{obs}}$. The in vitro data were fit to various kinetic models to derive the $\mathrm{K}_{\mathrm{I}}$ and $\mathrm{K}_{\text {inact }}$ values, and the model selected was dependent on the shape of the $\mathrm{k}_{\mathrm{obs}}$ versus concentration profile.

Of the 18 evaluated inhibitors, 16 demonstrated TDI in human hepatocytes, and kinetic parameters could be confidently derived for them. TDI toward CYP2C19 was not observed in hepatocytes for fluconazole or osilodrostat although reversible inhibition parameters could be derived for use in clinical risk assessment with basic models. Fluconazole is known to be a potent reversible inhibitor of CYP2C9, CYP2C19, and CYP3A. As TDI necessitates formation of a reactive metabolite, the lack of TDI by fluconazole is consistent with the knowledge that fluconazole is poorly metabolized and primarily eliminated unchanged via renal excretion (Bellmann and Smuszkiewicz, 2017). The lack of TDI observed for osilodrostat in hepatocytes was in contrast to data generated in HLM, where inactivation parameters could be derived (Armani et al., 2017). However, the DDI observed with omeprazole $\left(\right.$ AUCR $=1.91$ ) was well predicted using the estimated $K_{i}$ for reversible inhibition in the mechanistic static model (AUCR $=2.57$ ). Of note, there was no inhibition of CYP2C19 observed for moclobemide in hepatocytes despite a clinically relevant interaction with omeprazole $(\mathrm{AUCR}=2.07)$. Moclobemide has been reported to inhibit CYP2C19 in vitro, and TDI parameters were estimated based on clinical observations of autoinhibition (Kanacher et al., 2020).

Although CYP2B6 inhibitors were not investigated in the current study, due to limited available clinical data, TDI parameters derived in an alternate pool of human hepatocytes, for ticlopidine, were used to conduct clinical risk assessment for clinical data available with CYP2B6 substrates with the goal to evaluate whether the observations made for CYP2B6 were consistent with the other enzymes evaluated. In the enclosed studies, ticlopidine was included as a strong inhibitor toward CYP2C19 since there was clinical data demonstrating AUCR increases up to sixfold for omeprazole. Importantly, ticlopidine did not result in TDI of CYP2C19 in this hepatocyte donor pool; however, TDI kinetic parameters could be derived in the same donor pool as used for CYP2B6, and those were used to evaluate clinical risk predictions and for comparisons with data generated from HLM. It is unclear why there were differences observed between donor pools, although it's important to recognize that this variability exists in the in vitro model. There was limited data available for the selected inhibitors in HLM with only gemfibrozil, gemfibrozil glucuronide, omeprazole, osilodrostat, paroxetine, and ticlopidine having published data. There were no reported inactivation parameters for these inhibitors and enzymes using hepatocytes as the in vitro test system. In general, the available TDI parameters from hepatocytes were limited to CYP3A.

In recent examples, TDI data generated from HLM coupled with PBPK modeling has resulted in quantitative prediction of DDI potential consistent with clinical changes observed (Armani et al., 2017; Perkins et al., 2018; Tseng et al., 2021). While PBPK modeling was in scope for this project, it was not pursued given that quantitative predictions were observed when applying the MSM. Multiple laboratories have demonstrated improvement in DDI prediction accuracy using human hepatocytes suspended in human plasma particularly for CYP3A inhibitors (Lu et al., 2007; Lu et al., 2008; Mao et al., 2011). Despite the data supporting this observation for CYP3A, data available with nonCYP3A enzymes is lacking, thus plasma was not included in these studies with the aim to limit confounding factors. This is also consistent with the approach taken in a recent study where the authors compared TDI of CYP3A generated for 50 drugs in both HLM and hepatocytes to establish boundary values for $\mathrm{k}_{\mathrm{obs}}$, which would reduce the number of false positives observed in their screening assay (Eng et al., 2020). To understand whether the current basic equations recommended in the regulatory guidance documents, which are based on IVIVE for CYP3A, are applicable to TDI for non-CYP3A enzymes, analysis was conducted with the goal of: (1) establishing a multiplier to the " $R_{2}$ " equation, which would reduce the number of false positives and not result in increased false negatives and (2) establish a quantitative prediction model through investigation of various input parameters in the MSM.

When applying a 50-fold multiplier, as proposed in the $\mathrm{R}_{2}$ equation within the FDA, and PMDA regulatory guidance, a high rate of overpredictions was observed for data generated in both HHEPs and HLM. When the multiplier is removed, dronedarone became a false positive. It should be noted that dronedarone is highly lipophilic although less so than amiodarone (Hohnloser et al., 2009) which may have led to underestimation of the inactivation parameters from HHEP. When a multiplier of 3 was used, the false negatives were eliminated but the quantitative prediction was still poor ( $13 \%$ within twofold). When the mechanistic static model, which considers the substrate $F_{m}$, was used there was good alignment between the predicted DDI and observed DDI even when multiple substrates with varying $F_{m}$ values were used with the same inhibitor. When the unbound $\mathrm{C}_{\max , \mathrm{ss}}$ concentration was used rather than $\mathrm{I}_{\text {inlet,max, }}$, dronedarone was one false negative in HHEPs and there were a significant number of underpredictions (15/ 18). When the $I_{\text {inlet,max, }}$ was used as the input, along with inhibitor specific values for its derivation $\left(\mathrm{K}_{\mathrm{a}}, \mathrm{F}_{\mathrm{a}}, \mathrm{F}_{\mathrm{g}}\right.$, and $\left.\mathrm{R}_{\mathrm{b}}\right)$, there were no false negatives and $92 \%$ of the dataset was predicted within twofold of the observed values. Considering that there are situations where these values are unknown, default parameters of 0.03 for $\mathrm{K}_{\mathrm{a}}, 1$ for $\mathrm{F}_{\mathrm{a}}$ and $\mathrm{F}_{\mathrm{g}}$ and $R_{b}$ of 0.55 , were also evaluated and resulted in $91 \%$ of the studies being predicted within twofold. In summary, these studies revealed that quantitative IVIVE for CYP1A2, 2C8, 2C9, 2C19, and 2D6 inhibition is possible when using kinetic parameters generated in HHEPs.

A comparison between HHEPs and HLM was made for nine inhibitors where data were available in literature or generated in-house. It is important to note that of the 17 inhibitors evaluated, using standard HLM conditions, only four exhibited TDI. The reason for this discrepancy is unclear, although it is possible that some optimization of the incubation conditions, such as using a lower protein concentration, may enable TDI detection, as previously published for omeprazole (Ogilvie et al., 2011). Where comparisons were possible, there was a tendency for underprediction of DDI when using the data from HLM compared with HHEPs. This observation is in contrast to recent observations for CYP3A TDI (Tseng et al., 2021). The inactivation parameters generated from HHEP were almost always lower than those generated in HLMs for these non-CYP3A enzymes. This is similar to the observations made for CYP3A, where the authors hypothesized that there may be differences in the enzyme behavior in the intact cell model (Tseng et al., 2021). In that work, the authors further concluded that CYP3A TDI could be accurately predicted using the unbound average hepatic inlet concentration for gut and unbound average circulating concentration for liver, when using the MSM. When TDI inhibitor parameters for non-CYP3A enzymes were coupled with inhibitor and substrate specific 
parameters in the MSM, there were a number of $\mathrm{FN}$ and underpredictions observed when using the $\mathrm{C}_{\mathrm{max}, \mathrm{ss}, \mathrm{u}}$, and therefore evaluation of $\mathrm{C}_{\mathrm{avg}, \mathrm{ss}, \mathrm{u}}$ was not pursued. A notable difference between the assessment for CYP3A and non-CYP3A DDI is the importance of gut CYP3A in the overall magnitude of the observed DDI. Presumably the concentration used to project hepatic DDI would be consistent across CYP enzymes, suggesting that further optimization of the gut input is warranted for predicting CYP3A inhibition DDI.

Results from this study show that incorporating kinetic parameters for TDI into the previously proposed MSM enables quantitative prediction of TDI for CYPs 1A2, 2B6, 2C8, 2C9, 2C19, and 2D6. Additionally, analysis of the available HLM data also demonstrates reasonable quantitative prediction using the MSM, confirming that in vitro parameters derived from HLM are likewise valuable for TDI risk assessment from non-CYP3A enzymes, although the analysis would benefit from additional data points.

\section{Acknowledgements}

The authors thank prior and current management for their support of this collaborative project, including Jing-Tao Wu, Saeho Chong, Suresh Balani, Natalie Hosea, Mitchell Taub, Tom Chan, and Ting Wang. The authors would like to acknowledge Ritu Singh at the Broad Institute, previously employed at Corning, for her contributions to the initial design of the in vitro studies and review of the clinical data. The authors would also like to thank members of the ICH M12 guidance team, including Heidi Einolf, Ernesto Callegari, Scott Obach, Theunis Goosen, Elaine Lovering, Xinning Yang, and Carolien Versantvoort for helpful discussions on the data.

\section{Authorship Contributions}

Participated in research design: Ramsden, Perloff, Whitcher-Johnstone, Zhang.

Conducted experiments: Ho, Patel, Kozminski, Fullenwider.

Performed data analysis: Ramsden, Perloff, Whitcher-Johnstone, Kozminski, Fullenwider.

Wrote or contributed to the writing of the manuscript: Ramsden, Perloff, Whitcher-Johnstone, Zhang.

\section{References}

Armani S, Ting L, Sauter N, Darstein C, Tripathi AP, Wang L, Zhu B, Gu H, Chun DY, Einolf HJ, et al. (2017) Drug interaction potential of osilodrostat (LCI699) based on its effect on the pharmacokinetics of probe drugs of cytochrome P450 enzymes in healthy adults. Clin Drug Investig 37:465-472.

Backman JT, Honkalammi J, Neuvonen M, Kurkinen KJ, Tornio A, Niemi M, and Neuvonen PJ (2009) CYP2C8 activity recovers within 96 hours after gemfibrozil dosing: estimation of CYP2C8 half-life using repaglinide as an in vivo probe. Drug Metab Dispos 37:2359-2366.

Bellmann R and Smuszkiewicz P (2017) Pharmacokinetics of antifungal drugs: practical implications for optimized treatment of patients. Infection 45:737-779.

Brown HS, Wilby AJ, Alder J, and Houston JB (2010) Comparative use of isolated hepatocytes and hepatic microsomes for cytochrome P450 inhibition studies: transporter-enzyme interplay. Drug Metab Dispos 38:2139-2146.

Burt HJ, Neuhoff S, Almond L, Gaohua L, Harwood MD, Jamei M, Rostami-Hodjegan A, Tucker GT, and Rowland-Yeo K (2016) Metformin and cimetidine: physiologically based pharmacokinetic modelling to investigate transporter mediated drug-drug interactions. Eur J Pharm Sci 88:70-82.

Chen Y, Liu L, Monshouwer M, and Fretland AJ (2011) Determination of time-dependent inactivation of CYP3A4 in cryopreserved human hepatocytes and assessment of human drug-drug interactions. Drug Metab Dispos 39:2085-2092.

Chu X, Korzekwa K, Elsby R, Fenner K, Galetin A, Lai Y, Matsson P, Moss A, Nagar S, Rosania GR, et al.; International Transporter Consortium (2013) Intracellular drug concentrations and transporters: measurement, modeling, and implications for the liver. Clin Pharmacol Ther 94:126-141.

Djebli N, Fabre D, Boulenc X, Fabre G, Sultan E, and Hurbin F (2015) Physiologically based pharmacokinetic modeling for sequential metabolism: effect of CYP2C19 genetic polymorphism on clopidogrel and clopidogrel active metabolite pharmacokinetics. Drug Metab Dispos 43:510-522.

Eng H, Tseng E, Cerny MA, Goosen TC, and Obach RS (2020) Cytochrome P450 3A time-dependent inhibition assays are too sensitive for identification of drugs causing clinically significant drug-drug interactions: a comparison of human liver microsomes and hepatocytes and definition of boundaries for inactivation rate constants. Drug Metab Dispos 49:442-450.

Faber MS and Fuhr U (2004) Time response of cytochrome P450 1A2 activity on cessation of heavy smoking. Clin Pharmacol Ther 76:178-184.

Fahmi OA, Maurer TS, Kish M, Cardenas E, Boldt S, and Nettleton D (2008) A combined model for predicting CYP3A4 clinical net drug-drug interaction based on CYP3A4 inhibition, inactivation, and induction determined in vitro. Drug Metab Dispos 36:1698-1708.

Grimm SW, Einolf HJ, Hall SD, He K, Lim HK, Ling KH, Lu C, Nomeir AA, Seibert E, Skordos $\mathrm{KW}$, et al. (2009) The conduct of in vitro studies to address time-dependent inhibition of drug- metabolizing enzymes: a perspective of the pharmaceutical research and manufacturers of America. Drug Metab Dispos 37:1355-1370.

Hallifax D and Houston JB (2006) Binding of drugs to hepatic microsomes: comment and assessment of current prediction methodology with recommendation for improvement. Drug Metab Dispos 34:724-726, author reply 727.

Hohnloser SH, Crijns HJ, van Eickels M, Gaudin C, Page RL, Torp-Pedersen C, Connolly SJ; ATHENA Investigators (2009) Effect of dronedarone on cardiovascular events in atrial fibrillation. N Engl J Med 360:668-678.

Isoherranen N, Lutz JD, Chung SP, Hachad H, Levy RH, and Ragueneau-Majlessi I (2012) Importance of multi-p450 inhibition in drug-drug interactions: evaluation of incidence, inhibition magnitude, and prediction from in vitro data. Chem Res Toxicol 25:2285-2300.

Jogiraju VK, Heimbach T, Toderika Y, and Taft DR (2021) Physiologically based pharmacokinetic modeling of altered tizanidine systemic exposure by CYP1A2 modulation: impact of drugdrug interactions and cigarette consumption. Drug Metab Pharmacokinet 37:100375.

Kanacher T, Lindauer A, Mezzalana E, Michon I, Veau C, Mantilla JDG, Nock V, and Fleury A (2020) A physiologically-based pharmacokinetic (PBPK) model network for the prediction of CYP1A2 and CYP2C19 drug-drug-gene interactions with fluvoxamine, omeprazole, S-mephenytoin, moclobemide, tizanidine, mexiletine, ethinylestradiol, and caffeine. Pharmaceutics 12:1191.

Keefer C, Chang G, Carlo A, Novak JJ, Banker M, Carey J, Cianfrogna J, Eng H, Jagla C, Johnson $\mathrm{N}$, et al. (2020) Mechanistic insights on clearance and inhibition discordance between liver microsomes and hepatocytes when clearance in liver microsomes is higher than in hepatocytes. Eur J Pharm Sci 155:105541.

Kenny JR, Mukadam S, Zhang C, Tay S, Collins C, Galetin A, and Khojasteh SC (2012) Drugdrug interaction potential of marketed oncology drugs: in vitro assessment of time-dependent cytochrome P450 inhibition, reactive metabolite formation and drug-drug interaction prediction. Pharm Res 29:1960-1976.

Kilford PJ, Gertz M, Houston JB, and Galetin A (2008) Hepatocellular binding of drugs: correction for unbound fraction in hepatocyte incubations using microsomal binding or drug lipophilicity data. Drug Metab Dispos 36:1194-1197.

Kim SJ, Yoshikado T, Ieiri I, Maeda K, Kimura M, Irie S, Kusuhara H, and Sugiyama Y (2016) Clarification of the mechanism of clopidogrel-mediated drug-drug interaction in a clinical cassette small-dose study and its prediction based on in vitro information. Drug Metab Dispos 44:1622-1632.

Konishi K, Minematsu T, Nagasaka Y, and Tabata K (2019) Physiologically-based pharmacokinetic modeling for mirabegron: a multi-elimination pathway mediated by cytochrome P450 3A4, uridine 5'-diphosphate-glucuronosyltransferase 2B7, and butyrylcholinesterase. Xenobiotica 49:912-921.

Kostrubsky VE, Strom SC, Hanson J, Urda E, Rose K, Burliegh J, Zocharski P, Cai H, Sinclair JF, and Sahi J (2003) Evaluation of hepatotoxic potential of drugs by inhibition of bile-acid transport in cultured primary human hepatocytes and intact rats. Toxicol Sci 76:220-228.

Kurnik D, Wood AJ, and Wilkinson GR (2006) The erythromycin breath test reflects P-glycoprotein function independently of cytochrome P450 3A activity. Clin Pharmacol Ther 80:228-234.

Lam JL, Okochi H, Huang Y, and Benet LZ (2006) In vitro and in vivo correlation of hepatic transporter effects on erythromycin metabolism: characterizing the importance of transporterenzyme interplay. Drug Metab Dispos 34:1336-1344.

Leow JWH and Chan ECY (2019) Atypical Michaelis-Menten kinetics in cytochrome P450 enzymes: a focus on substrate inhibition. Biochem Pharmacol 169:113615.

Liston HL, DeVane CL, Boulton DW, Risch SC, Markowitz JS, and Goldman J (2002) Differential time course of cytochrome P450 2D6 enzyme inhibition by fluoxetine, sertraline, and paroxetine in healthy volunteers. J Clin Psychopharmacol 22:169-173.

Lu C, Hatsis P, Berg C, Lee FW, and Balani SK (2008) Prediction of pharmacokinetic drug-drug interactions using human hepatocyte suspension in plasma and cytochrome P450 phenotypic data. II. In vitro-in vivo correlation with ketoconazole. Drug Metab Dispos 36:1255-1260.

Lu C, Miwa GT, Prakash SR, Gan LS, and Balani SK (2007) A novel model for the prediction of drug-drug interactions in humans based on in vitro cytochrome p450 phenotypic data. Drug Metab Dispos 35:79-85.

Mao J, Mohutsky MA, Harrelson JP, Wrighton SA, and Hall SD (2011) Prediction of CYP3A-mediated drug-drug interactions using human hepatocytes suspended in human plasma. Drug Metab Dispos 39:591-602.

Marsousi N, Desmeules JA, Rudaz S, and Daali Y (2018) Prediction of drug-drug interactions using physiologically-based pharmacokinetic models of CYP450 modulators included in Simcyp software. Biopharm Drug Dispos 39:3-17.

Mateus A, Matsson P, and Artursson P (2013) Rapid measurement of intracellular unbound drug concentrations. Mol Pharm 10:2467-2478.

Miki A, Ohtani H, and Sawada Y (2011) Warfarin and miconazole oral gel interactions: analysis and therapy recommendations based on clinical data and a pharmacokinetic model. J Clin Pharm Ther 36:642-650.

Nagar S, Argikar UA, and Tweedie DJ (2014a) Enzyme kinetics in drug metabolism: fundamentals and applications. Methods Mol Biol 1113:1-6.

Nagar S, Jones JP, and Korzekwa K (2014b) A numerical method for analysis of in vitro timedependent inhibition data. Part 1. Theoretical considerations. Drug Metab Dispos 42:1575-1586.

Nishihara M, Ramsden D, and Balani SK (2021) Evaluation of the drug-drug interaction potential for trazpiroben (TAK-906), a $\mathrm{D}_{2} / \mathrm{D}_{3}$ receptor antagonist for gastroparesis, towards cytochrome P450s and transporters. Xenobiotica 51:668-679.

O'Reilly RA, Goulart DA, Kunze KL, Neal J, Gibaldi M, Eddy AC, and Trager WF (1992) Mechanisms of the stereoselective interaction between miconazole and racemic warfarin in human subjects. Clin Pharmacol Ther 51:656-667.

Obach RS, Walsky RL, and Venkatakrishnan K (2007) Mechanism-based inactivation of human cytochrome p450 enzymes and the prediction of drug-drug interactions. Drug Metab Dispos 35:246-255.

Ogilvie BW, Yerino P, Kazmi F, Buckley DB, Rostami-Hodjegan A, Paris BL, Toren P, and Parkinson A (2011) The proton pump inhibitor, omeprazole, but not lansoprazole or pantoprazole, is a metabolism-dependent inhibitor of CYP2C19: implications for coadministration with clopidogrel. Drug Metab Dispos 39:2020-2033.

Ogilvie BW, Zhang D, Li W, Rodrigues AD, Gipson AE, Holsapple J, Toren P, and Parkinson A (2006) Glucuronidation converts gemfibrozil to a potent, metabolism-dependent inhibitor of CYP2C8: implications for drug-drug interactions. Drug Metab Dispos 34:191-197.

Orr ST, Ripp SL, Ballard TE, Henderson JL, Scott DO, Obach RS, Sun H, and Kalgutkar AS (2012) Mechanism-based inactivation (MBI) of cytochrome P450 enzymes: structure-activity relationships and discovery strategies to mitigate drug-drug interaction risks. J Med Chem 55:4896-4933.

Paine MF, Hart HL, Ludington SS, Haining RL, Rettie AE, and Zeldin DC (2006) The human intestinal cytochrome P450 "pie". Drug Metab Dispos 34:880-886. 
Parkinson A, Kazmi F, Buckley DB, Yerino P, Ogilvie BW, and Paris BL (2010) System-dependent outcomes during the evaluation of drug candidates as inhibitors of cytochrome P450 (CYP) and uridine diphosphate glucuronosyltransferase (UGT) enzymes: human hepatocytes versus liver microsomes versus recombinant enzymes. Drug Metab Pharmacokinet 25:16-27.

Perkins EJ, Posada M, Kellie Turner P, Chappell J, Ng WT, and Twelves C (2018) Physiologically based pharmacokinetic modelling of cytochrome P450 2C9-related tolbutamide drug interactions with sulfaphenazole and tasisulam. Eur J Drug Metab Pharmacokinet 43:355-367.

Perloff ES, Mason AK, Dehal SS, Blanchard AP, Morgan L, Ho T, Dandeneau A, Crocker RM, Chandler CM, Boily N, et al. (2009) Validation of cytochrome P450 time-dependent inhibition assays: a two-time point IC50 shift approach facilitates kinact assay design. Xenobiotica 39:99-112.

Ramsden D, Fung C, Hariparsad N, Kenny JR, Mohutsky M, Parrott NJ, Robertson S, and Tweedie DJ (2019) Perspectives from the Innovation and Quality Consortium Induction Working Group on factors impacting clinical drug-drug interactions resulting from induction: focus on cytochrome 3A substrates. Drug Metab Dispos 47:1206-1221.

Ramsden D, Wallace G, Smith C, and Chan T (2009) A distribution analysis of cytochrome P450 Enzyme activities in cryopreserved human hepatocytes from over 400 individuals. ISSX $\mathbf{2 2 5}$.

Renwick AB, Watts PS, Edwards RJ, Barton PT, Guyonnet I, Price RJ, Tredger JM, Pelkonen O, Boobis AR, and Lake BG (2000) Differential maintenance of cytochrome P450 enzymes in cultured precision-cut human liver slices. Drug Metab Dispos 28:1202-1209.

Riccardi K, Li Z, Brown JA, Gorgoglione MF, Niosi M, Gosset J, Huard K, Erion DM, and Di L (2016) Determination of unbound partition coefficient and in vitro-in vivo extrapolation for SLC13A transporter-mediated uptake. Drug Metab Dispos 44:1633-1642.

Seelig A and Landwojtowicz E (2000) Structure-activity relationship of P-glycoprotein substrates and modifiers. Eur J Pharm Sci 12:31-40.

Thelen K and Dressman JB (2009) Cytochrome P450-mediated metabolism in the human gut wall. J Pharm Pharmacol 61:541-558.

Tornio A, Filppula AM, Kailari O, Neuvonen M, Nyrönen TH, Tapaninen T, Neuvonen PJ, Niemi M, and Backman JT (2014) Glucuronidation converts clopidogrel to a strong time-dependent inhibitor of CYP2C8: a phase II metabolite as a perpetrator of drug-drug interactions. Clin Pharmacol Ther 96:498-507.

Tseng E, Eng H, Lin J, Cerny MA, Tess DA, Goosen TC, and Obach RS (2021). Static and dynamic projections of drug-drug interactions caused by cytochrome P450 3A time-dependent inhibitors measured in human liver microsomes and hepatocytes. Drug Metab Dispos 49:947-960.
Varma MV, Lin J, Bi YA, Kimoto E, and Rodrigues AD (2015) Quantitative rationalization of gemfibrozil drug interactions: consideration of transporters-enzyme interplay and the role of circulating metabolite gemfibrozil 1-O- $\beta$-glucuronide. Drug Metab Dispos 43:1108-1118

Varma MV, Obach RS, Rotter C, Miller HR, Chang G, Steyn SJ, El-Kattan A, and Troutman MD (2010) Physicochemical space for optimum oral bioavailability: contribution of human intestinal absorption and first-pass elimination. J Med Chem 53:1098-1108.

Venkatakrishnan K and Obach RS (2005) In vitro-in vivo extrapolation of CYP2D6 inactivation by paroxetine: prediction of nonstationary pharmacokinetics and drug interaction magnitude. Drug Metab Dispos 33:845-852.

Vieira ML, Kirby B, Ragueneau-Majlessi I, Galetin A, Chien JY, Einolf HJ, Fahmi OA, Fischer V, Fretland A, Grime K, et al. (2014) Evaluation of various static in vitro-in vivo extrapolation models for risk assessment of the CYP3A inhibition potential of an investigational drug. Clin Pharmacol Ther 95:189-198.

Xie F, Ding X, and Zhang QY (2016) An update on the role of intestinal cytochrome P450 enzymes in drug disposition. Acta Pharm Sin B 6:374-383.

Xu L, Chen Y, Pan Y, Skiles GL, and Shou M (2009) Prediction of human drug-drug interactions from time-dependent inactivation of CYP3A4 in primary hepatocytes using a population-based simulator. Drug Metab Dispos 37:2330-2339.

Xu RJ, Kong WM, An XF, Zou JJ, Liu L, and Liu XD (2020) Physiologically-based pharmacokinetic-pharmacodynamics model characterizing CYP2C19 polymorphisms to predict clopidogrel pharmacokinetics and its anti-platelet aggregation effect following oral administration to coronary artery disease patients with or without diabetes. Front Pharmacol 11:593982.

Yamada M, Inoue SI, Sugiyama D, Nishiya Y, Ishizuka T, Watanabe A, Watanabe K, Yamashita S, and Watanabe N (2020) Critical impact of drug-drug interactions via intestinal CYP3A in the risk assessment of weak perpetrators using physiologically based pharmacokinetic models. Drug Metab Dispos 48:288-296.

Zetterberg C, Maltais F, Laitinen L, Liao S, Tsao H, Chakilam A, and Hariparsad N (2016) VX509 (decernotinib)-mediated CYP3A time-dependent inhibition: an aldehyde oxidase metabolite as a perpetrator of drug-drug interactions. Drug Metab Dispos 44:1286-1295.x

Address correspondence to: Diane Ramsden, 35 Gatehouse Park, Waltham, MA 02451. E-mail: diane.ramsden@takeda.com; or Elke S. Perloff, 6 Henshaw Street, Woburn, MA 01801. E-mail: perloffe@ corning.com 